\title{
Light pseudoscalar meson and heavy meson scattering lengths to $\mathcal{O}\left(p^{4}\right)$ in heavy meson chiral perturbation theory
}

\author{
Bo-Lin Huang $\oplus^{*}$ Zi-Yang Lin, ${ }^{\dagger}$ and Shi-Lin Zhu $\oplus^{*}$ \\ School of Physics and Center of High Energy Physics, Peking University, Beijing 100871, China
}

(Received 29 December 2021; accepted 2 February 2022; published 25 February 2022)

\begin{abstract}
We calculate the threshold $T$ matrices of the light pseudoscalar meson and heavy meson scattering to fourth order in heavy meson chiral perturbation theory. We determine the low-energy constants by fitting to the lattice QCD data points through both the perturbative and iterated methods and obtain the physical scattering lengths in both formalisms. The values of the scattering lengths tend to be convergent at fourth order for most of the channels in the perturbative method. The value of the scattering length for the channel $D K(I=0)$, which involves the bound state $D_{s 0}^{*}(2317)$, is obtained correctly in the iterated method. Based on the heavy diquark-antiquark symmetry, we also estimate the meson and doubly charmed (bottom) baryon scattering lengths, and find that the bound states can be generated with high probability in the channels $\bar{K} \Xi_{c c}(I=0)$ and $\bar{K} \Xi_{b b}(I=0)$. We strongly urge the LHCb Collaboration to look for the very narrow $\bar{K} \Xi_{c c}$ state with $I J^{P}=0 \frac{1}{2}$ through either the electromagnetic decay or the iso-spin violating strong decay $\Omega_{c c} \pi$.
\end{abstract}

DOI: 10.1103/PhysRevD.105.036016

\section{INTRODUCTION}

Since the discovery of the charm-strange meson $D_{s 0}^{*}(2317)$ with $J^{P}=0^{+}[1-3]$, many investigations have been devoted to this puzzling state because its mass lies significantly below the quark model predictions [4-7]. The low mass puzzle of the $D_{s 0}^{*}(2317)$ inspired various explanations, which include the $D^{(*)} K$ molecular [8-11], the $c q \bar{s} \bar{q}$ tetraquark state [12-15], the conventional charmedstrange mesons with coupled-channel effects [16], and the lattice QCD simulations [17-22] (for a detailed review see Ref. [23]). In Ref. [20], the low-energy interactions between light pseudoscalar mesons and charmed pseudoscalar mesons were studied. The $S$-wave scattering lengths of the $D \bar{K}(I=0,1), D_{s} K, D \pi(I=3 / 2)$, and $D_{s} \pi$ were calculated using Lüscher's finite volume technique in full lattice QCD. The scattering lengths of the $D \pi(I=1 / 2)$, $D K(I=1,0)$, and $D_{s} K$ at the physical pion mass were predicted. The attraction in the channel $D K(I=0)$ is so strong that a pole can be generated, and the result supported the interpretation of the $D_{s 0}^{*}(2317)$ as a $D K$ molecule. Since the $D_{s 0}^{*}(2317)$ couples to the $D K$ channel strongly,

\footnotetext{
*blhuang@pku.edu.cn

†1zy_15@pku.edu.cn

zhusl@pku.edu.cn
}

Published by the American Physical Society under the terms of the Creative Commons Attribution 4.0 International license. Further distribution of this work must maintain attribution to the author(s) and the published article's title, journal citation, and DOI. Funded by SCOAP ${ }^{3}$. the detailed study of the S-wave $D K$ scattering will help us understand the nature of this exotic state.

The scattering length is an important observable in the scattering process, which encodes the information of the fundamental interaction and can be extracted from the threshold $T$ matrix. It is a popular method to obtain the $T$ matrix from the chiral perturbation theory (ChPT). However, the low-energy constants in the chiral Lagrangian need to be determined from the experimental data. It is a good choice to take the lattice QCD data for the light pseudoscalar meson and heavy meson interaction from Refs. [19-22,24-26] when the experimental data is scanty. In lattice calculations, the light pseudoscalar meson masses are always larger than their physical masses. Therefore, the extrapolation of the scattering length from the nonphysical meson mass to the physical value is necessary with the help of ChPT.

ChPT is a useful and efficient tool to study the hadronic physics at low energies. Based on Weinberg's powercounting $[27,28]$, the chiral expressions can be organized by the power of the small external momentum or the meson momentum (or mass). The heavy baryon chiral perturbation theory (HBChPT) was proposed and developed to solve the power-counting problem which occurs in baryon ChPT [29-31]. Many achievements have been obtained in SU(2) HBChPT [32-39]. From Refs. [40-47], it turned out that the calculations in SU(3) HBChPT can also lead to reasonable predictions.

Similar to the HBChPT formalism in the meson-baryon interaction, we use heavy meson chiral perturbation theory (HMChPT) to deal with the charmed mesons [48]. In this framework, the heavy meson $D$ is nonrelativistic. 
However, the $1 / M$ corrections disappear when the threshold amplitudes are considered in HMChPT. Thus, there is no difference when the scattering lengths are either calculated in the HMChPT or the covariant ChPT (for a review of these approaches, see Ref. [49]).

In our previous paper [50], we have calculated the light pseudoscalar meson and heavy meson $S$-wave scattering lengths up to $\mathcal{O}\left(p^{3}\right)$ in HMChPT and obtained a positive scattering length for the channel $D K(I=0)$. Note that, a repulsive interaction has a negative scattering length in our convention. Therefore, the channel $D K(I=0)$ is attractive. However, the $T$ matrix would not produce a pole in the perturbative calculation, which corresponds to a bound state or resonance. Thus, the perturbative scattering length for the channel $D K(I=0)$ would not turn into a negative one. In fact, the channel $D K(I=0)$ with a bound state $\left[D_{s 0}^{*}(2317)\right]$ has a strong enough attractive interaction, and can lead to a negative scattering length. Then, the calculations with various iterated methods are performed in Refs. [20,51-56]. They all obtained a negative scattering length for the channel $D K(I=0)$ based on the amplitudes below order $\mathcal{O}\left(p^{4}\right)$. Nevertheless, an attractive interaction can lead to either a positive scattering length or a negative one. Thus, the perturbative calculation for the scattering length is also necessary. In this paper, we will calculate the threshold $T$ matrices of the light pseudoscalar meson and heavy meson scattering to $\mathcal{O}\left(p^{4}\right)$ in HMChPT in order to obtain a more precise perturbative result. Then, we will also use the iterated method to calculate the scattering length in order to obtain the correct scattering length for the channel which may admit a bound state or resonance. At last, we estimate the mesons and doubly charmed (bottom) baryon scattering lengths based on the heavy diquark-antiquark symmetry.

This paper is organized as follows. In Sec. II, we present the chiral Lagrangians up to $\mathcal{O}\left(p^{4}\right)$. In Sec. III, we present the Feynman diagrams and results of the threshold $T$ matrices. In Sec. IV, we outline how to derive scattering lengths from the $T$ matrices. Section $\mathrm{V}$ contains the presentation and discussion of our results. The last section is a brief summary. Appendixes A and B contain the various scattering lengths of the light pseudoscalar meson and doubly charmed (bottom) baryons, respectively.

\section{CHIRAL LAGRANGIAN}

In order to calculate the light pseudoscalar meson and heavy meson scattering lengths up to order $\mathcal{O}\left(p^{4}\right)$ in heavy meson chiral perturbation theory, the corresponding effective Lagrangian can be written as

$$
\mathcal{L}_{\text {eff }}=\mathcal{L}_{\phi \phi}^{(2)}+\mathcal{L}_{H \phi}^{(1)}+\mathcal{L}_{H \phi}^{(2)}+\mathcal{L}_{H \phi}^{(3)}+\mathcal{L}_{H \phi}^{(4)} .
$$

The traceless Hermitian $3 \times 3$ matrices $\phi$ include the pseudoscalar Goldstone boson fields $(\pi, K, \bar{K}, \eta)$. The lowest-order SU(3) chiral Lagrangian for the Goldstone meson-meson interaction take the form [57]

$$
\mathcal{L}_{\phi \phi}^{(2)}=f^{2} \operatorname{tr}\left(u_{\mu} u^{\mu}+\frac{\chi_{+}}{4}\right),
$$

where $f$ is the pseudoscalar decay constant in the chiral limit. The axial vector quantity $u^{\mu}=\frac{i}{2}\left\{\xi^{\dagger}, \partial^{\mu} \xi\right\}$ contains an odd number of meson fields. The quantity $\chi_{+}=\xi^{\dagger} \chi \xi^{\dagger}+\xi \chi \xi$ with $\chi=\operatorname{diag}\left(m_{\pi}^{2}, m_{\pi}^{2}, 2 m_{K}^{2}-m_{\pi}^{2}\right)$ introduces explicit chiral symmetry breaking terms. We choose the $\mathrm{SU}(3)$ matrix

$$
U=\xi^{2}=\exp (i \phi / f),
$$

which collects the pseudoscalar Goldstone boson fields.

The lowest-order chiral Lagrangian for the heavy mesons in the heavy quark symmetry limit is

$\mathcal{L}_{H \phi}^{(1)}=-\langle(i v \cdot \partial H) \bar{H}\rangle+\langle H v \cdot \Gamma \bar{H}\rangle+g\left\langle H u_{\mu} \gamma^{\mu} \gamma_{5} \bar{H}\right\rangle$,

where $v_{\mu}=(1,0,0,0)$ is the heavy meson velocity, the chiral connection $\Gamma^{\mu}=\frac{i}{2}\left[\xi^{\dagger}, \partial^{\mu} \xi\right]$ contains an even number of meson fields and the doublet of the ground state heavy mesons reads

$$
\begin{gathered}
H=\frac{1+\not p}{2}\left(P_{\mu}^{*} \gamma^{\mu}+i P \gamma_{5}\right), \quad \bar{H}=\gamma^{0} H^{\dagger} \gamma^{0}=\left(P_{\mu}^{* \dagger} \gamma^{\mu}+i P^{\dagger} \gamma_{5}\right) \frac{1+\not p}{2}, \\
P=\left(D^{0}, D^{+}, D_{s}^{+}\right), \quad P_{\mu}^{*}=\left(D^{0 *}, D^{+*}, D_{s}^{+*}\right)_{\mu} .
\end{gathered}
$$

For the calculation of the threshold $T$ matrices, the heavy meson Lagrangians $\mathcal{L}_{H \phi}^{(2)}, \mathcal{L}_{H \phi}^{(3)}$, and $\mathcal{L}_{H \phi}^{(4)}$ in the heavy quark symmetry limit read

$$
\begin{gathered}
\mathcal{L}_{H \phi}^{(2)}=c_{0}\langle H \bar{H}\rangle \operatorname{tr}\left(\chi_{+}\right)+c_{1}\left\langle H \chi_{+} \bar{H}\right\rangle-c_{2}\langle H \bar{H}\rangle \operatorname{tr}(v \cdot u v \cdot u)-c_{3}\langle H v \cdot u v \cdot u \bar{H}\rangle \\
-c_{4}\langle H \bar{H}\rangle \operatorname{tr}\left(u^{\mu} u_{\mu}\right)-c_{5}\left\langle H u^{\mu} u_{\mu} \bar{H}\right\rangle, \\
\mathcal{L}_{H \phi}^{(3)}=\kappa\left\langle H\left[\chi_{-}, v \cdot u\right] \bar{H}\right\rangle,
\end{gathered}
$$




$$
\mathcal{L}_{H \phi}^{(4)}=e_{1}\langle H \bar{H}\rangle \operatorname{tr}[(v \cdot \partial v \cdot u)(v \cdot \partial v \cdot u)]+e_{2}\langle H(v \cdot \partial v \cdot u)(v \cdot \partial v \cdot u) \bar{H}\rangle
$$

Here, the terms with quark mass in $\mathcal{L}_{H \phi}^{(4)}$ are not considered explicitly. Indeed, for the quark mass terms, some of the dimension four low-energy constants (LECs) simply amount to quark mass renormalizations of some of the dimension two $c_{i}$. Thus, the contributions from the quark mass terms can be absorbed into the dimension two LECs. We can neglect the quark-mass terms in our calculation. The predictions for the scattering lengths will not be affected. This is a very general phenomenon of ChPT calculations in higher orders (for details, see, e.g., Refs. [34,57]).

\section{THRESHOLD $T$ MATRICES}

We present the explicit expressions for the threshold $T$ matrices to $\mathcal{O}\left(p^{4}\right)$ in the chiral expansion. In order to obtain a more compact representation for the $T$ matrices, the four subfunctions are introduced,

$$
\begin{gathered}
L\left(m_{1}, m_{2}\right)=\sqrt{m_{1}^{2}-m_{2}^{2}} \ln \frac{m_{1}+\sqrt{m_{1}^{2}-m_{2}^{2}}}{m_{2}}, \\
L_{i}\left(m_{1}, m_{2}\right)=\sqrt{m_{1}^{2}-m_{2}^{2}}\left(i \pi-\ln \frac{m_{1}+\sqrt{m_{1}^{2}-m_{2}^{2}}}{m_{2}}\right), \\
A\left(m_{1}, m_{2}\right)=\sqrt{m_{2}^{2}-m_{1}^{2}} \arccos \frac{m_{1}}{m_{2}} \\
M(\alpha, \beta, \gamma)=\alpha m_{K}^{4}+\beta m_{K}^{2} m_{\pi}^{2}+\gamma m_{\pi}^{4} .
\end{gathered}
$$

Here, the $L_{i}\left(m_{1}, m_{2}\right)$ contains the imaginary part and comes from the iterated diagrams $(\mathrm{g}),(\mathrm{m})$, and $(\mathrm{n})$ of Fig. 1. The leading-order (LO), next-to-leading order (NLO), next-to-next-to-leading order (N2LO), and nextto-next-to-next-to-leading order (N3LO) $T$ matrices are from the diagrams (a), (b), (c), and (d) in Fig. 1, respectively. At N2LO and N3LO, we have the contributions from the one-loop diagrams (e)-(j) and (k)-(r) of Fig. 1, which involve the vertices from the Lagrangians $\mathcal{L}_{H \phi}^{(1)}$ and $\mathcal{L}_{H \phi}^{(2)}$, respectively. The contributions from the vector charmed mesons will not be considered explicitly in this paper. The influence of the $D^{*}$ on the scattering lengths may be marginal [55]. The description for the S-wave scattering lengths is expected to be a good approximation in the calculation. In the following $T$ matrices of the elastic light pseudoscalar meson and heavy meson scattering, we use $K=\left(K^{+}, K^{0}\right)^{T}$ and $\bar{K}=\left(\bar{K}^{0}, K^{-}\right)^{T}$. The superscripts of the $T$ matrices denote the total isospin. In the channels with an isoscalar $\eta$-meson or $D_{s}$-meson, the total isospin is unique and does not need to be specified. We take the renormalized (nonzero quark mass) decay constants $f_{\pi, K, \eta}$

instead of $f$ (the chiral limit value, zero quark mass). For the construction of the one-loop and the counterterm $T$ matrics, the scale-independent LECs $\bar{\kappa}, \bar{e}_{1}$, and $\bar{e}_{2}$ are used. Thus, all terms $\ln \left(m_{\pi, K, \eta} / \mu\right)$ have disappeared in our final expressions. The Gell-Mann-Okubo (GMO) relation $m_{\eta}=$ $\sqrt{\left(4 m_{K}^{2}-m_{\pi}^{2}\right) / 3}$ has also been used to simplify the equations. The $c_{2}$ and $c_{4}\left(c_{3}\right.$ and $\left.c_{5}\right)$ terms are different at N3LO only. They are strongly correlated with each other. However, we will keep all of them because the prediction of the physical quantity will not be affected when the correlations are considered. Then, the $T$ matrices with the four orders read

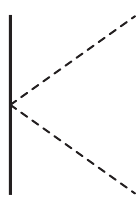

(a)

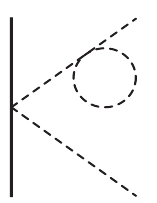

(e)

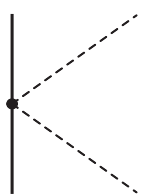

(b)

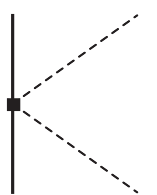

(c)

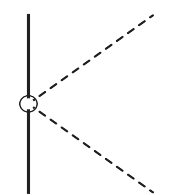

(d)

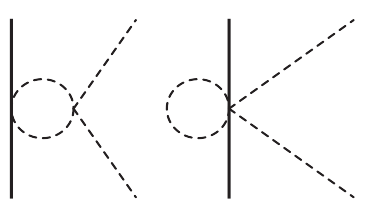

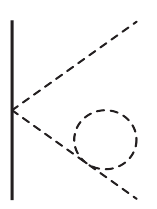

(f)

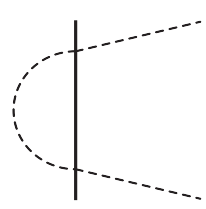

(g)

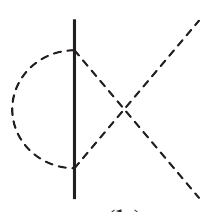

(h) (i)

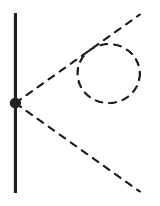

(k) (j)

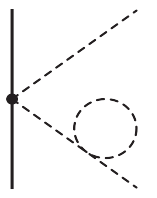

(1)

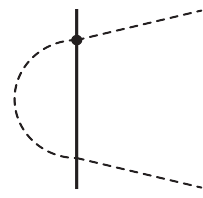

(m)

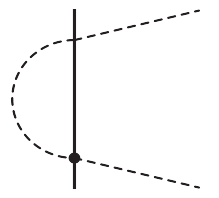

(n)<smiles>CC12CCC(C1)C[I-]2(C)C</smiles>

(o)

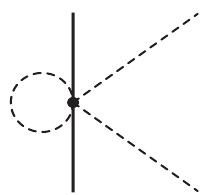

(p)

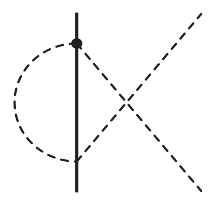

(q)

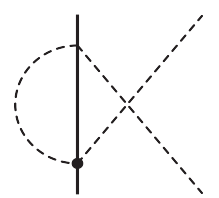

(r)
FIG. 1. Tree and nonvanishing loop diagrams in the calculation of meson-heavy meson scattering lengths to the fourth order in HMChPT. Dashed lines represent Goldstone bosons and solid lines represent pseudoscalar heavy mesons. The heavy dots, filled squares, and open circles refer to vertices from $\mathcal{L}_{H \phi}^{(2)}, \mathcal{L}_{H \phi}^{(3)}$, and $\mathcal{L}_{H \phi}^{(4)}$, respectively. 


$$
\begin{aligned}
& T_{D K}^{(1)}=\{0\}_{\mathrm{LO}}+\left\{\frac{2\left(4 c_{0}+c_{2}+c_{4}\right) m_{K}^{2}}{f_{K}^{2}}\right\}_{\mathrm{NLO}}+\left\{\frac{m_{K}^{2}}{8 \pi^{2} f_{K}^{4}} L_{i}\left(m_{K}, m_{\pi}\right)\right\}_{\mathrm{N} 2 \mathrm{LO}}+\left\{-\frac{2 \bar{e}_{1} m_{K}^{4}}{f_{K}^{2}}\right. \\
& +\frac{1}{6912 \pi^{2} f_{K}^{4}}\left\{192 c_{0} M(40,-5,7)-96 c_{1}\left[M(48,5,-5)+36 m_{K}\left(m_{\pi}^{2}+m_{K}^{2}\right) L_{i}\left(m_{K}, m_{\pi}\right)\right]\right. \\
& -6 c_{2} M(1,-8,7)-c_{3}\left[M(1753,-8,19)+1728 m_{K}^{3} L_{i}\left(m_{K}, m_{\pi}\right)\right]+48 c_{4} M(40,-5,7) \\
& \left.\left.+8 c_{5}\left[M(-170,7,19)-216 m_{K}^{3} L_{i}\left(m_{K}, m_{\pi}\right)\right]\right\}\right\}_{\mathrm{N} 3 \mathrm{LO}}, \\
& T_{D K}^{(0)}=\left\{\frac{2 m_{K}}{f_{K}^{2}}\right\}_{\mathrm{LO}}+\left\{\frac{2\left(4 c_{0}+4 c_{1}+c_{2}+c_{3}+c_{4}+c_{5}\right) m_{K}^{2}}{f_{K}^{2}}\right\}_{\mathrm{NLO}} \\
& +\left\{\frac{16 \bar{\kappa} m_{K}^{3}}{f_{K}^{2}}+\frac{3 m_{K}^{2}}{8 \pi^{2} f_{K}^{4}}\left[m_{K}-A\left(-m_{K}, m_{\eta}\right)\right]\right\}_{\mathrm{N} 2 \mathrm{LO}} \\
& +\left\{-\frac{2\left(\bar{e}_{1}+\bar{e}_{2}\right) m_{K}^{4}}{f_{K}^{2}}+\frac{1}{6912 \pi^{2} f_{K}^{4}}\left\{192 c_{0} M(40,-5,7)+96 c_{1}[M(204,-41,5)\right.\right. \\
& \left.-36 m_{K}\left(5 m_{K}^{2}-3 m_{\pi}^{2}\right) A\left(-m_{K}, m_{\eta}\right)\right]-6 c_{2} M(1,-8,7)+c_{3}\left[M(3557,8,-19)-1728 m_{K}^{3} A\left(-m_{K}, m_{\eta}\right)\right] \\
& \left.\left.+48 c_{4} M(40,-5,7)+8 c_{5}\left[M(478,7,19)-216 m_{K}^{3} A\left(-m_{K}, m_{\eta}\right)\right]\right\}\right\}_{\mathrm{N} 3 \mathrm{LO}}, \\
& T_{D \bar{K}}^{(1)}=\left\{-\frac{m_{K}}{f_{K}^{2}}\right\}_{\mathrm{LO}}+\left\{\frac{\left(8 c_{0}+4 c_{1}+2 c_{2}+c_{3}+2 c_{4}+c_{5}\right) m_{K}^{2}}{f_{K}^{2}}\right\}_{\mathrm{NLO}}+\left\{-\frac{8 \bar{\kappa} m_{K}^{3}}{f_{K}^{2}}+\frac{m_{K}^{2}}{16 \pi^{2} f_{K}^{4}}\left[-3 m_{K}\right.\right. \\
& \left.\left.-3 A\left(m_{K}, m_{\eta}\right)+L\left(m_{K}, m_{\pi}\right)\right]\right\}_{\mathrm{N} 2 \mathrm{LO}}+\left\{-\frac{\left(2 \bar{e}_{1}+\bar{e}_{2}\right) m_{K}^{4}}{f_{K}^{2}}+\frac{1}{6912 \pi^{2} f_{K}^{4}}\left\{192 c_{0} M(40,-5,7)\right.\right. \\
& +96 c_{1}\left[M(78,-23,5)+18 m_{K}\left(5 m_{K}^{2}-3 m_{\pi}^{2}\right) A\left(m_{K}, m_{\eta}\right)+18 m_{K}\left(m_{K}^{2}+m_{\pi}^{2}\right) L\left(m_{K}, m_{\pi}\right)\right] \\
& -6 c_{2} M(1,-8,7)-c_{3}\left[M(902,8,-19)+864 m_{K}^{3} A\left(m_{K}, m_{\eta}\right)+864 m_{K}^{3} L\left(m_{K}, m_{\pi}\right)\right] \\
& \left.\left.+48 c_{4} M(40,-5,7)+8 c_{5}\left[M(154,7,19)+108 m_{K}^{3} A\left(m_{K}, m_{\eta}\right)+108 m_{K}^{3} L\left(m_{K}, m_{\pi}\right)\right]\right\}\right\}_{\mathrm{N} 3 \mathrm{LO}}, \\
& T_{D \bar{K}}^{(0)}=\left\{\frac{m_{K}}{f_{K}^{2}}\right\}_{\mathrm{LO}}+\left\{\frac{\left(8 c_{0}-4 c_{1}+2 c_{2}-c_{3}+2 c_{4}-c_{5}\right) m_{K}^{2}}{f_{K}^{2}}\right\}_{\mathrm{NLO}}+\left\{\frac{8 \bar{\kappa} m_{K}^{3}}{f_{K}^{2}}+\frac{3 m_{K}^{2}}{16 \pi f_{K}^{4}}\left[m_{K}\right.\right. \\
& \left.\left.+A\left(m_{K}, m_{\eta}\right)+L\left(m_{K}, m_{\pi}\right)\right]\right\}_{\mathrm{N} 2 \mathrm{LO}}+\left\{\frac{\left(-2 \bar{e}_{1}+\bar{e}_{2}\right) m_{K}^{4}}{f_{K}^{2}}+\frac{1}{6912 \pi^{2} f_{K}^{4}}\left\{192 c_{0} M(40,-5,7)\right.\right. \\
& +96 c_{1}\left[M(-174,13,5)+m_{K}\left(-90 m_{K}^{2}+54 m_{\pi}^{2}\right) A\left(m_{K}, m_{\eta}\right)+54\left(m_{K}^{2}+m_{\pi}^{2}\right) L\left(m_{K}, m_{\pi}\right)\right] \\
& -6 c_{2} M(1,-8,7)-c_{3}\left[M(4408,-8,19)+864 m_{K}^{3} A\left(m_{K}, m_{\eta}\right)-2592 m_{K}^{3} L\left(m_{K}, m_{\pi}\right)\right] \\
& \left.\left.+48 c_{4} M(40,-5,7)+8 c_{5}\left[M(-494,7,19)-108 m_{K}^{3} A\left(m_{K}, m_{\eta}\right)+324 m_{K}^{3} L\left(m_{K}, m_{\eta}\right)\right]\right\}\right\}_{\mathrm{N} 3 \mathrm{LO}}, \\
& T_{D_{s} K}=\left\{-\frac{m_{K}}{f_{K}^{2}}\right\}_{\mathrm{LO}}+\left\{\frac{\left(8 c_{0}+4 c_{1}+2 c_{2}+c_{3}+2 c_{4}+c_{5}\right) m_{K}^{2}}{f_{K}^{2}}\right\}_{\mathrm{NLO}}+\left\{-\frac{8 \bar{\kappa} m_{K}^{3}}{f_{K}^{2}}-\frac{3 m_{K}^{2}}{16 \pi^{2} f_{K}^{4}}\left[m_{K}\right.\right. \\
& \left.\left.+A\left(m_{K}, m_{\eta}\right)-L\left(m_{K}, m_{\pi}\right)\right]\right\}_{\mathrm{N} 2 \mathrm{LO}}+\left\{-\frac{\left(2 \bar{e}_{1}+\bar{e}_{2}\right) m_{K}^{4}}{f_{K}^{2}}+\frac{1}{6912 \pi^{2} f_{K}^{4}}\left\{192 c_{0} M(40,-5,7)\right.\right. \\
& +192 c_{1}\left[M(25,9,2)-9 m_{K}\left(5 m_{K}^{2}-3 m_{\pi}^{2}\right) A\left(m_{K}, m_{\eta}\right)-27 m_{K}\left(m_{K}^{2}+m_{\pi}^{2}\right) L\left(m_{K}, m_{\pi}\right)\right] \\
& -6 c_{2} M(1,-8,7)-c_{3}\left[M(-845,-32,4)+864 m_{K}^{3} A\left(m_{K}, m_{\eta}\right)+2592 m_{K}^{3} L\left(m_{K}, m_{\pi}\right)\right] \\
& \left.\left.+48 c_{4} M(40,-5,7)+32 c_{5}\left[M(64,-11,1)-27 m_{K}^{3} A\left(m_{K}, m_{\eta}\right)-81 m_{K}^{3} L\left(m_{K}, m_{\pi}\right)\right]\right\}\right\}_{\mathrm{N} 3 \mathrm{LO}},
\end{aligned}
$$




$$
\begin{aligned}
& T_{D_{s} \bar{K}}=\left\{\frac{m_{K}}{f_{K}^{2}}\right\}_{\mathrm{LO}}+\left\{\frac{\left(8 c_{0}+4 c_{1}+2 c_{2}+c_{3}+2 c_{4}+c_{5}\right) m_{K}^{2}}{f_{K}^{2}}\right\}_{\mathrm{NLO}}+\left\{\frac{8 \bar{\kappa} m_{K}^{3}}{f_{K}^{2}}-\frac{3 m_{K}^{2}}{16 \pi^{2} f_{K}^{4}}\left[m_{K}\right.\right. \\
& \left.\left.-A\left(-m_{K}, m_{\eta}\right)+L_{i}\left(m_{K}, m_{\pi}\right)\right]\right\}_{\mathrm{N} 2 \mathrm{LO}}+\left\{-\frac{\left(2 \bar{e}_{1}+\bar{e}_{2}\right) m_{K}^{4}}{f_{K}^{2}}+\frac{1}{6912 \pi^{2} f_{K}^{4}}\left\{192 c_{0} M(40,-5,7)\right.\right. \\
& +192 c_{1}\left[M(25,9,2)+9 m_{K}\left(5 m_{K}^{2}-3 m_{\pi}^{2}\right) A\left(-m_{K}, m_{\eta}\right)+27 m_{K}\left(m_{K}^{2}+m_{\pi}^{2}\right) L_{i}\left(m_{K}, m_{\pi}\right)\right] \\
& -6 c_{2} M(1,-8,7)+c_{3}\left[M(845,32,-4)+864 m_{K}^{3} A\left(-m_{K}, m_{\eta}\right)+2592 m_{K}^{3} L_{i}\left(m_{K}, m_{\pi}\right)\right] \\
& \left.\left.+48 c_{4} M(40,-5,7)+32 c_{5}\left[M(64,-11,1)+27 m_{K}^{3} A\left(-m_{K}, m_{\eta}\right)+81 m_{K}^{3} L_{i}\left(m_{K}, m_{\pi}\right)\right]\right\}\right\}_{\mathrm{N} 3 \mathrm{LO}}, \\
& T_{D \pi}^{(3 / 2)}=\left\{-\frac{m_{\pi}}{f_{\pi}^{2}}\right\}_{\mathrm{LO}}+\left\{\frac{\left(8 c_{0}+4 c_{1}+2 c_{2}+c_{3}+2 c_{4}+c_{5}\right) m_{\pi}^{2}}{f_{\pi}^{2}}\right\}_{\mathrm{NLO}}+\left\{-\frac{8 \bar{\kappa} m_{\pi}^{3}}{f_{\pi}^{2}}-\frac{m_{\pi}^{2}}{16 \pi^{2} f_{\pi}^{4}}\left[3 m_{\pi}\right.\right. \\
& \left.\left.+2 A\left(m_{\pi}, m_{K}\right)\right]\right\}_{\mathrm{N} 2 \mathrm{LO}}+\left\{-\frac{\left(2 \bar{e}_{1}+\bar{e}_{2}\right) m_{\pi}^{4}}{f_{\pi}^{2}}+\frac{1}{768 \pi^{2} f_{\pi}^{4}}\left\{128 c_{0} M(1,1,5)+32 c_{1}[M(1,7,16)\right.\right. \\
& \left.+12 m_{\pi}\left(m_{\pi}^{2}+m_{K}^{2}\right) A\left(m_{\pi}, m_{K}\right)\right]+4 c_{2}\left(m_{\pi}^{4}-m_{K}^{4}\right)-c_{3}\left[m_{K}^{4}-98 m_{\pi}^{4}-192 m_{\pi}^{3} A\left(m_{\pi}, m_{K}\right)\right] \\
& \left.\left.+32 c_{4} M(1,1,5)+8 c_{5}\left[M(1,1,22)+24 m_{\pi}^{3} A\left(m_{\pi}, m_{K}\right)\right]\right\}\right\}_{\mathrm{N} 3 \mathrm{LO}}, \\
& T_{D \pi}^{(1 / 2)}=\left\{\frac{2 m_{\pi}}{f_{\pi}^{2}}\right\}_{\mathrm{LO}}+\left\{\frac{\left(8 c_{0}+4 c_{1}+2 c_{2}+c_{3}+2 c_{4}+c_{5}\right) m_{\pi}^{2}}{f_{\pi}^{2}}\right\}_{\mathrm{NLO}}+\left\{\frac{16 \bar{\kappa} m_{\pi}^{3}}{f_{\pi}^{2}}-\frac{m_{\pi}^{2}}{16 \pi^{2} f_{\pi}^{4}}\left[6 m_{\pi}\right.\right. \\
& \left.\left.+A\left(m_{\pi}, m_{K}\right)-3 A\left(-m_{\pi}, m_{K}\right)\right]\right\}_{\mathrm{N} 2 \mathrm{LO}}+\left\{-\frac{\left(2 \bar{e}_{1}+\bar{e}_{2}\right) m_{\pi}^{4}}{f_{\pi}^{2}}+\frac{1}{768 \pi^{2} f_{\pi}^{4}}\left\{128 c_{0} M(1,1,5)\right.\right. \\
& +32 c_{1}\left[M(1,7,16)-6 m_{\pi}\left(m_{\pi}^{2}+m_{K}^{2}\right) A\left(m_{\pi}, m_{K}\right)-18 m_{\pi}\left(m_{\pi}^{2}+m_{K}^{2}\right) A\left(-m_{\pi}, m_{K}\right)\right] \\
& +4 c_{2}\left(m_{\pi}^{4}-m_{K}^{4}\right)-c_{3}\left[m_{K}^{4}-98 m_{\pi}^{4}+96 m_{\pi}^{3} A\left(m_{\pi}, m_{K}\right)+288 m_{\pi}^{3} A\left(-m_{\pi}, m_{K}\right)\right] \\
& \left.\left.+32 c_{4} M(1,1,5)+8 c_{5}\left[M(1,1,22)-12 m_{\pi}^{3} A\left(m_{\pi}, m_{K}\right)-36 m_{\pi}^{3} A\left(-m_{\pi}, m_{K}\right)\right]\right\}\right\}_{\mathrm{N} 3 \mathrm{LO}}, \\
& T_{D_{s} \pi}=\{0\}_{\mathrm{LO}}+\left\{\frac{2\left(4 c_{0}+c_{2}+c_{4}\right) m_{\pi}^{2}}{f_{\pi}^{2}}\right\}_{\mathrm{NLO}}+\left\{-\frac{1}{8 \pi^{2} f_{\pi}^{4}} m_{\pi}^{2}\left[A\left(-m_{\pi}, m_{K}\right)+A\left(m_{\pi}, m_{K}\right)\right]\right\}_{\mathrm{N} 2 \mathrm{LO}} \\
& +\left\{-\frac{2 \bar{e}_{1} m_{\pi}^{4}}{f_{\pi}^{2}}+\frac{1}{384 \pi^{2} f_{\pi}^{4}}\left\{64 c_{0} M(1,1,5)+32 c_{1}\left(m_{\pi}^{2}+m_{K}^{2}\right)\left[m_{K}^{2}-6 m_{\pi}^{2}\right.\right.\right. \\
& \left.+6 m_{\pi} A\left(-m_{\pi}, m_{K}\right)-6 m_{\pi} A\left(m_{\pi}, m_{K}\right)\right]+2 c_{2}\left(m_{\pi}^{4}-m_{K}^{4}\right)-c_{3}\left[m_{K}^{4}+96 m_{\pi}^{4}\right. \\
& \left.+96 m_{\pi}^{3} A\left(m_{\pi}, m_{K}\right)-96 m_{\pi}^{3} A\left(-m_{\pi}, m_{K}\right)\right]+16 c_{4} M(1,1,5)+8 c_{5}[M(1,1,-12) \\
& \left.\left.\left.+12 m_{\pi}^{3} A\left(-m_{\pi}^{2}, m_{K}\right)-12 m_{\pi}^{3} A\left(m_{\pi}, m_{K}\right)\right]\right\}\right\}_{\mathrm{N} 3 \mathrm{LO}}, \\
& T_{D \eta}=\{0\}_{\mathrm{LO}}+\left\{\frac{1}{9 f_{\eta}^{2}}\left[4\left(24 c_{0}+6 c_{2}+c_{3}+6 c_{4}+c_{5}\right) m_{K}^{2}-\left(24 c_{0}-12 c_{1}+6 c_{2}+c_{3}+6 c_{4}+c_{5}\right) m_{\pi}^{2}\right]\right\}_{\mathrm{NLO}} \\
& +\left\{\frac{3}{16 \pi f_{\eta}^{4}} m_{\eta}^{2}\left[L\left(m_{\eta}, m_{K}\right)+L_{i}\left(m_{\eta}, m_{K}\right)\right]\right\}_{\mathrm{N} 2 \mathrm{LO}}+\left\{\frac { 1 } { 2 5 6 \pi ^ { 2 } f _ { \eta } ^ { 4 } } \left\{128 c_{0} m_{K}^{2}\left(m_{\eta}^{2}+m_{K}^{2}\right)\right.\right. \\
& +32 c_{1}\left[-9 m_{\eta}^{2} m_{K}^{2}+6 m_{\eta}^{2} m_{\pi}^{2}+m_{K}^{4}+2 m_{\eta}\left(5 m_{K}^{2}-3 m_{\pi}^{2}\right) L\left(m_{\eta}, m_{K}\right)+2 m_{\eta}\left(-5 m_{K}^{2}\right.\right. \\
& \left.\left.+3 m_{\pi}^{2}\right) L_{i}\left(m_{\eta}, m_{K}\right)\right]-4 c_{2} m_{K}^{4}-c_{3}\left[32 m_{\eta}^{4}+m_{K}^{4}+32 L_{i}\left(m_{\eta}, m_{K}\right)-32 L\left(m_{\eta}, m_{K}\right)\right] \\
& +32 c_{4} m_{K}^{2}\left(m_{K}^{2}+m_{\eta}^{2}\right)+8 c_{5}\left[m_{K}^{4}+m_{K}^{2} m_{\eta}^{2}-4 m_{\eta}^{4}-4 m_{\eta}^{3} L\left(m_{\eta}, m_{K}\right)\right. \\
& \left.\left.\left.-4 m_{\eta}^{3} L_{i}\left(m_{\eta}, m_{K}\right)\right]\right\}\right\}_{\mathrm{N} 3 \mathrm{LO}}
\end{aligned}
$$




$$
\begin{aligned}
T_{D_{s} \eta}= & \{0\}_{\mathrm{LO}}+\left\{\frac { 1 } { 9 f _ { \eta } ^ { 2 } } \left[8\left(12 c_{0}+12 c_{1}+3 c_{2}+2 c_{3}+3 c_{4}+2 c_{5}\right) m_{K}^{2}-2\left(12 c_{0}+24 c_{1}+3 c_{2}+2 c_{3}\right.\right.\right. \\
& \left.\left.\left.+3 c_{4}+2 c_{5}\right) m_{\pi}^{2}\right]\right\}_{\mathrm{NLO}}+\left\{\frac{3}{8 \pi f_{\eta}^{4}} m_{\eta}^{2}\left[L\left(m_{\eta}, m_{K}\right)+L_{i}\left(m_{\eta}, m_{K}\right)\right]\right\}_{\mathrm{N} 2 \mathrm{LO}} \\
& +\left\{\frac { 1 } { 1 2 8 \pi ^ { 2 } f _ { \eta } ^ { 4 } } \left\{64 c_{0} m_{K}^{2}\left(m_{K}^{2}+m_{\eta}^{2}\right)+32 c_{1}\left[11 m_{\eta}^{2} m_{K}^{2}-6 m_{\eta}^{2} m_{\pi}^{2}+m_{K}^{4}\right.\right.\right. \\
& \left.\left.+2 m_{\eta}\left(5 m_{K}^{2}-3 m_{\pi}^{2}\right) L_{i}\left(m_{\eta}, m_{K}\right)-2 m_{\eta}\left(5 m_{K}^{2}-3 m_{\pi}^{2}\right) L_{(} m_{\eta}, m_{K}\right)\right]-2 c_{2} m_{K}^{4} \\
& -c_{3}\left[-32 m_{\eta}^{4}+m_{K}^{4}-32 m_{\eta}^{3} L_{i}\left(m_{\eta}, m_{K}\right)+32 m_{\eta}^{3} L\left(m_{\eta}, m_{K}\right)\right]+16 c_{4} m_{K}^{2}\left(m_{\eta}^{2}+m_{K}^{2}\right) \\
& \left.\left.+8 c_{5}\left[m_{K}^{4}+m_{K}^{2} m_{\eta}^{2}+4 m_{\eta}^{4}+4 m_{\eta}^{3} L_{i}\left(m_{\eta}, m_{K}\right)-4 m_{\eta}^{3} L\left(m_{\eta}, m_{K}\right)\right]\right\}\right\}_{\mathrm{N} 3 \mathrm{LO}}
\end{aligned}
$$

\section{SCATTERING LENGTHS}

The S-wave scattering length is defined through

$$
a=\frac{M}{8 \pi(M+m)} T_{\mathrm{th}},
$$

where $m$ and $M$ denote the light and heavy meson mass, respectively. We take the sign convention that a repulsive interaction has a negative scattering length. In order to obtain a correct description for the channels which may include bound states or resonances, the $T$ matrix must be iterated to infinite order. We can use a Lippmann-Schwinger equation with a cutoff range scale, denoted by $\mu$, to obtain a finite result, as done in Ref. [58]. For a single channel separable potential, the scattering length is given by

$$
a=a_{\mathrm{Born}}\left(1-\frac{1}{2} \mu a_{\mathrm{Born}}\right)^{-1} .
$$

Here $a_{\text {Born }}$ includes the contributions from all the diagrams except for the iterated diagrams (g), (m), and (n) of Fig. 1 in this work. The contributions from the three diagrams can be obtained through the iteration of the diagrams (a) and (b). Thus, our calculations include all the contributions from the tree diagrams, the renormalization diagrams, and the crossed diagrams up to forth order in the iteration of the potential. As a result, the scattering lengths will not include explicitly the imaginary parts.

\section{RESULTS AND DISCUSSION}

Before making predictions, we have to determine the lowenergy constants. We have the values $c_{0}=0.0045 \mathrm{GeV}^{-1}$ and $c_{1}=0.1112 \mathrm{GeV}^{-1}$ from Ref. [55] through the relations $h_{0}=2 M_{0} c_{0}$ and $h_{1}=2 M_{0} c_{1}$, where $M_{0}=1918 \mathrm{MeV}$ is taken for the charmed system [59]. For the other LECs, we have two fitting strategies to determine the pertinent constants. One is using the perturbative scattering length formula, Eq. (25), and the other is using the iterated scattering length formula, Eq. (26). For the perturbative formula, the $T$ matrices are from the Eqs. (14)-(24), and for the iterated formula, the contributions from the iterated diagrams $(\mathrm{g})$, (m), and (n) of Fig. 1 should be subtracted.

\section{A. Perturbative fitting}

Now we determine $c_{2,3,4,5}, \bar{\kappa}, \bar{e}_{1}$, and $\bar{e}_{2}$ using the perturbative formula, Eq. (25) and the lattice data of the five channels $[D \bar{K}(I=0), D \bar{K}(I=1), D \pi(I=3 / 2)$, $\left.D_{s} K, D_{s} \pi\right]$ which include the values of the scattering lengths and the masses of the pion, kaon, $D$, and $D_{s}$ mesons from Ref. [20], and the GMO relation is used throughout this paper. The corresponding lattice values of $f_{\pi}$ and $f_{K}$ are used from Ref. [24], and we always choose $f_{\eta}=1.2 f_{\pi}$ in this paper. The lattice data are obtained by using the unphysical quark mass, then the mass of the light meson can achieve a large value $(\sim 700 \mathrm{MeV})$. However, the chiral expressions are expanded in terms of $m_{\pi} / \Lambda_{\chi}$, where $m_{\pi}$ denotes the mass of the light meson and $\Lambda_{\chi}$ is the chiral symmetry breaking scale $(\sim 1 \mathrm{GeV})$. The large mass of the light meson maybe the cause of the problem of the convergence. Besides, the prediction for the scattering length should be independent of the inputs. Therefore, we take three different fits denoted as Fit p1, Fit p2, and Fit p3 which include the (M007, M010), (M007, M010, M020), and (M007, M010, M020, M030) data from Refs. [20,24], respectively. Thus, there are 10, 15, and 20 data in total for Fit p1, Fit p2, and Fit p3, respectively. The resulting LECs with the correlations between the parameters for the three fits can be found in Table I. The values of the LECs from Fit p1 and Fit p2 are roughly consistent, and the resulting LECs from Fit $\mathrm{p} 3$ are the large differences because the large mass of the light meson are taken. However, the results from Fit p3 are still valid because the light meson mass is still smaller than the chiral symmetry break scale $(\sim 1 \mathrm{GeV})$. The uncertainty for the respective parameter is statistical, and it measures how 
TABLE I. Values of the various fits with the correlations between the parameters through the perturbative scattering lengths formula, Eq. (25). For a detailed description of these fits, see the main text.

\begin{tabular}{|c|c|c|c|c|c|c|c|c|}
\hline & Fit p1 & $c_{2}$ & $c_{3}$ & $c_{4}$ & $c_{5}$ & $\bar{\kappa}$ & $\bar{e}_{1}$ & $\bar{e}_{2}$ \\
\hline$c_{2}\left(\mathrm{GeV}^{-1}\right)$ & $0.53 \pm 3.12$ & 1.00 & -0.83 & -0.99 & 0.83 & 0.90 & -0.25 & -0.73 \\
\hline$c_{3}\left(\mathrm{GeV}^{-1}\right)$ & $-2.82 \pm 3.59$ & & 1.00 & 0.90 & -0.99 & -0.86 & 0.67 & 0.49 \\
\hline$c_{4}\left(\mathrm{GeV}^{-1}\right)$ & $-0.26 \pm 2.71$ & & & 1.00 & -0.90 & -0.91 & 0.38 & 0.69 \\
\hline$c_{5}\left(\mathrm{GeV}^{-1}\right)$ & $3.22 \pm 4.44$ & & & & 1.00 & 0.86 & -0.67 & -0.49 \\
\hline $\bar{\kappa}\left(\mathrm{GeV}^{-2}\right)$ & $0.87 \pm 0.51$ & & & & & 1.00 & -0.33 & -0.85 \\
\hline $\bar{e}_{1}\left(\mathrm{GeV}^{-3}\right)$ & $-0.44 \pm 1.30$ & & & & & & 1.00 & -0.18 \\
\hline $\bar{e}_{2}\left(\mathrm{GeV}^{-3}\right)$ & $-6.15 \pm 4.54$ & & & & & & & 1.00 \\
\hline$\chi^{2} /$ d.o.f. & $\frac{3.41}{10-7}=1.14$ & & & & & & & \\
\hline
\end{tabular}

\begin{tabular}{|c|c|c|c|c|c|c|c|c|}
\hline & Fit p2 & $c_{2}$ & $c_{3}$ & $c_{4}$ & $c_{5}$ & $\bar{\kappa}$ & $\bar{e}_{1}$ & $\bar{e}_{2}$ \\
\hline$c_{2}\left(\mathrm{GeV}^{-1}\right)$ & $0.96 \pm 2.02$ & 1.00 & -0.76 & -0.99 & 0.77 & 0.81 & -0.44 & -0.53 \\
\hline$c_{3}\left(\mathrm{GeV}^{-1}\right)$ & $-3.46 \pm 1.84$ & & 1.00 & 0.84 & -0.99 & -0.70 & 0.86 & 0.21 \\
\hline$c_{4}\left(\mathrm{GeV}^{-1}\right)$ & $-0.63 \pm 1.68$ & & & 1.00 & -0.84 & -0.82 & 0.55 & 0.48 \\
\hline$c_{5}\left(\mathrm{GeV}^{-1}\right)$ & $4.08 \pm 2.28$ & & & & 1.00 & 0.71 & -0.86 & -0.22 \\
\hline $\bar{\kappa}\left(\mathrm{GeV}^{-2}\right)$ & $0.98 \pm 0.29$ & & & & & 1.00 & -0.41 & -0.84 \\
\hline $\bar{e}_{1}\left(\mathrm{GeV}^{-3}\right)$ & $-0.05 \pm 0.66$ & & & & & & 1.00 & -0.11 \\
\hline $\bar{e}_{2}\left(\mathrm{GeV}^{-3}\right)$ & $-7.62 \pm 2.65$ & & & & & & & 1.00 \\
\hline$\chi^{2} /$ d.o.f. & $\frac{10.43}{15-7}=1.30$ & & & & & & & \\
\hline
\end{tabular}

\begin{tabular}{|c|c|c|c|c|c|c|c|c|}
\hline & Fit p3 & $c_{2}$ & $c_{3}$ & $c_{4}$ & $c_{5}$ & $\bar{\kappa}$ & $\bar{e}_{1}$ & $\bar{e}_{2}$ \\
\hline$c_{2}\left(\mathrm{GeV}^{-1}\right)$ & $-1.05 \pm 1.15$ & 1.00 & -0.69 & -0.99 & 0.71 & 0.78 & -0.66 & -0.45 \\
\hline$c_{3}\left(\mathrm{GeV}^{-1}\right)$ & $-0.34 \pm 0.83$ & & 1.00 & 0.76 & -0.99 & -0.51 & 0.89 & 0.03 \\
\hline$c_{4}\left(\mathrm{GeV}^{-1}\right)$ & $1.34 \pm 0.93$ & & & 1.00 & -0.79 & -0.76 & 0.74 & 0.40 \\
\hline$c_{5}\left(\mathrm{GeV}^{-1}\right)$ & $0.20 \pm 1.03$ & & & & 1.00 & 0.54 & -0.90 & -0.05 \\
\hline $\bar{\kappa}\left(\mathrm{GeV}^{-2}\right)$ & $0.54 \pm 0.17$ & & & & & 1.00 & -0.43 & -0.85 \\
\hline $\bar{e}_{1}\left(\mathrm{GeV}^{-3}\right)$ & $0.99 \pm 0.35$ & & & & & & 1.00 & -0.07 \\
\hline $\bar{e}_{2}\left(\mathrm{GeV}^{-3}\right)$ & $-5.35 \pm 1.62$ & & & & & & & 1.00 \\
\hline$\chi^{2} /$ d.o.f. & $\frac{21.76}{20-7}=1.67$ & & & & & & & \\
\hline
\end{tabular}

much a particular parameter can be changed while maintaining a good description of the fitted data. However, the LECs cannot really vary independently of each other because of the mutual correlations, as detailed in Refs. $[60,61]$. Thus, the large uncertainties for LECs in our fits will not make the errors of the scattering lengths become large, because a full error analysis requires a complete covariance matrix which indicates the mutual correlations between the parameters. One of the reasons for the large uncertainties is that the number of data is small but the number of parameters is large. We can find that the errors of Fit $\mathrm{p} 3$ are smaller than the ones of Fit p1. The other reason is that the LECs correlate each other, especially for the $c_{2}$ and $c_{4}\left(c_{3}\right.$ and $\left.c_{5}\right)$. The absolute value of the correlation between $c_{2}$ and $c_{4}\left(c_{3}\right.$ and $\left.c_{5}\right)$ is very close to one (0.99), which is consistent with the fact that the difference of the $T$ matrix only appears at N3LO. Therefore, we can remove one of them. However, all of them are still retained in our calculation because the prediction of the scattering length will not be affected when the correlations are considered. Besides the large uncertainties and the strong correlations, the values of the LECs are of natural size. The result is consistent with the assumption that the contributions from the $D^{*}$ vector mesons are marginal. We can also find that the absolute value of $c_{2}\left(c_{4}\right)$ is much smaller than $c_{3}\left(c_{5}\right)$ in Fit p1 and Fit $\mathrm{p} 2$ because the $c_{2}\left(c_{4}\right)$ term is suppressed by $1 / N_{c}$ as compared to the $c_{3}\left(c_{5}\right)$ term. In Fit $\mathrm{p} 3$, the feature is inverse because the large masses of the light pseudoscalar mesons reduce the fitting accuracy.

The corresponding scattering lengths from the three fits are shown in Fig. 2. The scattering lengths of the five channels except for the $D \bar{K}(I=1)$ from all of the three fits are in good agreement with the data from lattice QCD when $m_{\pi} \simeq 301,364 \mathrm{MeV}$. For the $D \bar{K}(I=1)$, the value of the scattering length from lattice QCD has large error $(\sim 34 \%)$ at $m_{\pi} \simeq 301 \mathrm{MeV}$. However, the channel at this pion mass only has a small repulsive interaction $(\sim-0.14 \mathrm{fm})$ which is far away from the nonlinear region generated by the pole of the iterated scattering length formula. Thus, the large error for the data point should have a small influence on the prediction for the strong attractive interaction. For Fit p1, 

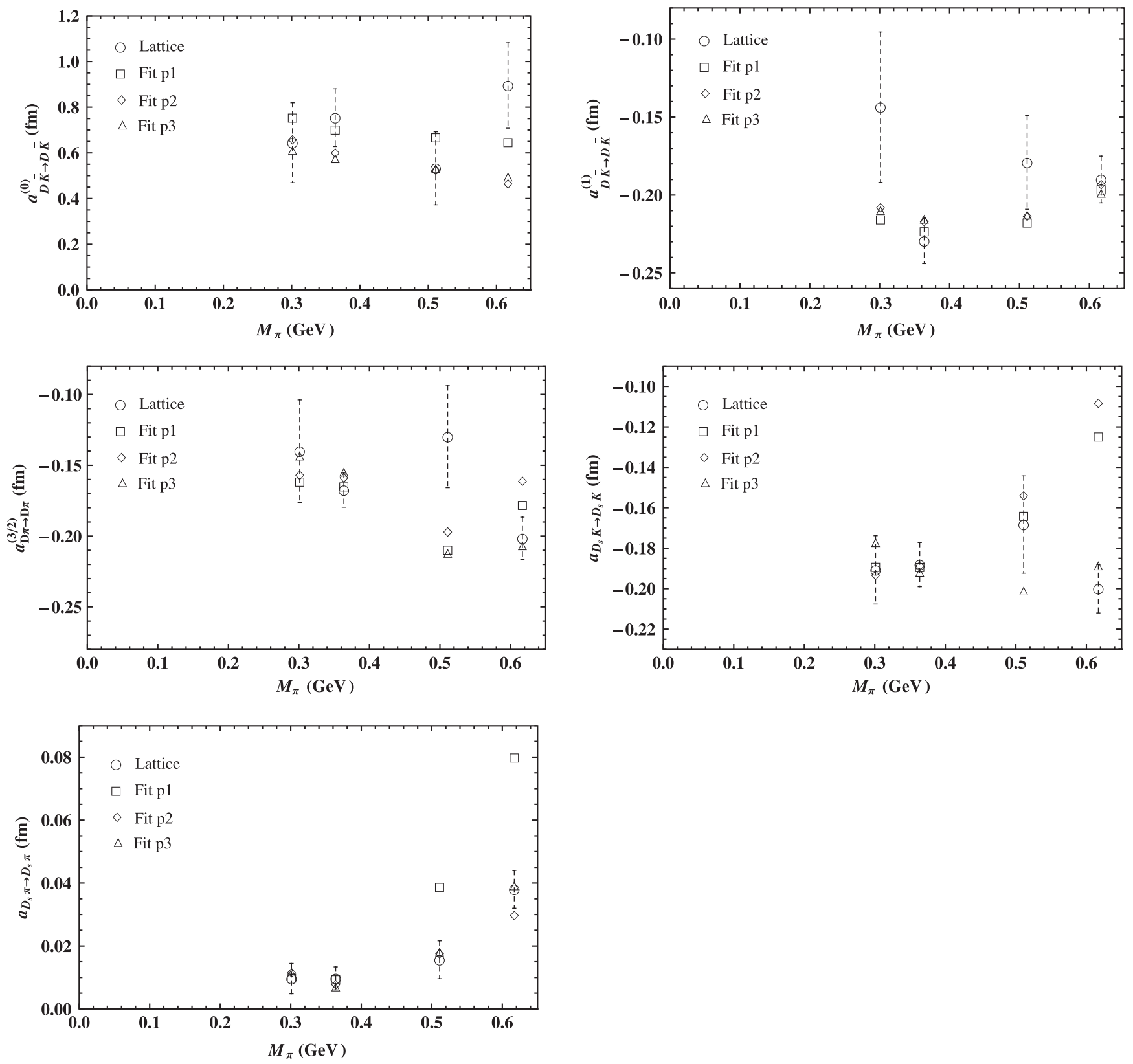

FIG. 2. Comparison of the results of the five-channel fits through the perturbative formula, Eq. (25), to the lattice data of the scattering lengths from Ref. [20].

the predictions of the scattering lengths at $m_{\pi} \simeq$ $511,617 \mathrm{MeV}$ are away from the lattice QCD in most data points, but they still have the same order of magnitude. For Fit $\mathrm{p} 2$ and $\mathrm{p} 3$, the descriptions of the scattering lengths at $m_{\pi} \simeq 511,617 \mathrm{MeV}$ have been improved, but the deviations are significantly larger than the ones at the smaller mass of pion. The chiral expansions for the scattering lengths are expanded in terms of the $m_{\pi, K, \eta} / \Lambda$. Therefore, the large mass of the light pseudoscalar meson causes the poor convergence and reduces the reliability of the predictions of the scattering lengths. Nevertheless, the values of the extrapolation from the low mass to high mass are consistent with the results from lattice QCD in the same order of magnitude.

In the following, we make predictions of the scattering lengths for the 11 channels at the physical values using the LECs determined above. We use the values of the physical parameters: $m_{\pi}=139.57 \mathrm{MeV}, m_{K}=493.68 \mathrm{MeV}$, $f_{\pi}=92.07 \mathrm{MeV}, f_{K}=110.03 \mathrm{MeV}, M_{D}=1869.66 \mathrm{MeV}$, $M_{D s}=1968.35 \mathrm{MeV}$ from PDG [62]. The results with the three different fits are shown in Table II. The errors of the scattering lengths in the total values are estimated from the statistical errors of the LECs using the error propagation formula with the correlations. We can see that the errors 
TABLE II. Predictions of the scattering lengths through the perturbative formula, Eq. (25). The LECs are from the three different perturbative fits, see Table I. The scattering lengths are in units of fm.

\begin{tabular}{lccccc}
\hline \hline Fit $\mathrm{p} 1$ & $\mathcal{O}(p)$ & $\mathcal{O}\left(p^{2}\right)$ & $\mathcal{O}\left(p^{3}\right)$ & $\mathcal{O}\left(p^{4}\right)$ & Total \\
\hline$a_{D K}^{(1)}$ & 0.00 & 0.07 & $-0.12+0.19 i$ & $0.09-0.13 i$ & $0.04(3)+0.07(16) i$ \\
$a_{D K}^{(0)}$ & 0.51 & 0.29 & 0.77 & 0.41 & $1.98(93)$ \\
$a_{D \bar{K}}^{(1)}$ & -0.25 & 0.18 & -0.49 & 0.33 & $-0.23(3)$ \\
$a_{D \bar{K}}^{(0)}$ & 0.25 & -0.04 & 0.73 & -0.16 & $0.80(6)$ \\
$a_{D_{s} K}$ & -0.26 & 0.18 & -0.38 & 0.24 & $-0.21(7)$ \\
$a_{D_{s} \bar{K}}$ & 0.26 & 0.18 & $0.21+0.29 i$ & $0.33+0.19 i$ & $0.97(47)+0.49(22) i$ \\
$a_{D \pi}^{(3 / 2)}$ & -0.12 & 0.02 & -0.04 & 0.04 & $-0.09(3)$ \\
$a_{D \pi}^{(1 / 2)}$ & 0.24 & 0.02 & 0.02 & -0.01 & $0.27(2)$ \\
$a_{D_{s} \pi}$ & 0.00 & 0.01 & -0.04 & 0.05 & $0.02(2)$ \\
$a_{D \eta}$ & 0.00 & 0.11 & $0.00+0.21 i$ & $0.02-0.10 i$ & $0.13(8)+0.11(3) i$ \\
$a_{D_{s} \eta}$ & 0.00 & 0.32 & $0.00+0.43 i$ & $0.18+0.20 i$ & $0.50(26)+0.62(13) i$ \\
\hline \hline
\end{tabular}

\begin{tabular}{|c|c|c|c|c|c|}
\hline Fit $\mathrm{p} 2$ & & & & & \\
\hline$\overline{a_{D K}^{(1)}}$ & 0.00 & 0.09 & $-0.12+0.19 i$ & $0.06-0.16 i$ & $0.03(3)+0.03(8) i$ \\
\hline$a_{D K}^{(0)}$ & 0.51 & 0.35 & 0.87 & 0.47 & $2.20(52)$ \\
\hline$a_{D \bar{K}}^{(1)}$ & -0.25 & 0.22 & -0.55 & 0.36 & $-0.22(2)$ \\
\hline$a_{D \bar{K}}^{(0)}$ & 0.25 & -0.05 & 0.79 & -0.23 & $0.76(6)$ \\
\hline $\begin{array}{c}D K \\
a_{D_{s} K}\end{array}$ & -0.26 & 0.22 & -0.43 & 0.24 & $-0.22(4)$ \\
\hline$a_{D_{\bar{k}} \bar{K}}$ & 0.26 & 0.22 & $0.26+0.29 i$ & $0.34+0.25 i$ & $1.07(27)+0.54(10) t$ \\
\hline$a_{D-}^{(3 / 2)}$ & -0.12 & 0.03 & -0.04 & 0.03 & $-0.09(2)$ \\
\hline$a_{D \pi}^{(1 / 2)}$ & 0.24 & 0.03 & 0.02 & -0.02 & $0.27(2)$ \\
\hline$a_{D_{s} \pi}$ & 0.00 & 0.01 & -0.04 & 0.05 & $0.02(2)$ \\
\hline$a_{D \eta}$ & 0.00 & 0.14 & $0.00+0.21 i$ & $0.00-0.11 i$ & $0.14(5)+0.10(1) i$ \\
\hline$a_{D_{s} \eta}$ & 0.00 & 0.38 & $0.00+0.43 i$ & $0.19+0.23 i$ & $0.57(15)+0.65(7) i$ \\
\hline
\end{tabular}

\begin{tabular}{lrcccc}
\hline \hline Fit $\mathrm{p} 3$ & & & & & \\
\hline$a_{D K}^{(1)}$ & 0.00 & 0.08 & $-0.12+0.19 i$ & $0.04-0.02 i$ & $-0.00(2)+0.17(4) i$ \\
$a_{D K}^{(0)}$ & 0.51 & 0.16 & 0.44 & 0.34 & $1.44(30)$ \\
$a_{D \bar{K}}^{(1)}$ & -0.25 & 0.12 & -0.33 & 0.24 & $-0.22(2)$ \\
$a_{D \bar{K}}^{(0)}$ & 0.25 & 0.04 & 0.57 & -0.16 & $0.70(4)$ \\
$a_{D_{s} K}$ & -0.26 & 0.12 & -0.21 & 0.20 & $-0.15(2)$ \\
$a_{D_{s} \bar{K}}$ & 0.26 & 0.12 & $0.04+0.29 i$ & $0.25+0.03 i$ & $0.66(15)+0.33(4) i$ \\
$a_{D \pi}^{(3 / 2)}$ & -0.12 & 0.02 & -0.03 & 0.06 & $-0.07(2)$ \\
$a_{D \pi}^{(1 / 2)}$ & 0.24 & 0.02 & 0.01 & 0.02 & $0.28(1)$ \\
$a_{D_{s} \pi}$ & 0.00 & 0.01 & -0.04 & 0.05 & $0.03(1)$ \\
$a_{D \eta}$ & 0.00 & 0.09 & $0.00+0.21 i$ & $0.09-0.05 i$ & $0.19(3)+0.16(0) i$ \\
$a_{D_{s} \eta}$ & 0.00 & 0.21 & $0.00+0.43 i$ & $0.16+0.11 i$ & $0.37(8)+0.53(3) i$ \\
\hline \hline
\end{tabular}

from the LECs with large errors are not too large to be unacceptable because of the correlations between the parameters. The errors in the respective orders are not given because we do not fit the scattering lengths up to the corresponding orders, and the values of the scattering lengths from the respective orders (i.e., the contributions exclude the lower orders) are presented. We can find that the results tend to be convergent for most of the channels in the three fits. The results from the three fits are almost consistent, and the large difference does not occur. The reason is that the formulas for the predictions are linear, and the results are not very sensitive to the inputs. There exist only weak attractions or weak repulsions in most channels. We find that the channel $D K(I=0)$ has strong attraction but still not strong enough to generate the well-known bound state $D_{s 0}^{*}(2317)$. The value of the scattering length 
TABLE III. Values of the various fits with the correlations between the parameters through the iterated scattering lengths formula, Eq. (26). For a detailed description of these fits, see the main text.

\begin{tabular}{|c|c|c|c|c|c|c|c|c|c|}
\hline & Fit u1 & $c_{2}$ & $c_{3}$ & $c_{4}$ & $c_{5}$ & $\bar{\kappa}$ & $\bar{e}_{1}$ & $\bar{e}_{2}$ & $\mu$ \\
\hline$c_{2}\left(\mathrm{GeV}^{-1}\right)$ & $-4.60 \pm 4.58$ & 1.00 & -0.92 & -0.99 & 0.89 & 0.92 & 0.70 & 0.75 & 0.19 \\
\hline$c_{3}\left(\mathrm{GeV}^{-1}\right)$ & $3.32 \pm 3.42$ & & 1.00 & 0.94 & -0.98 & -0.87 & -0.57 & -0.71 & -0.10 \\
\hline$c_{4}\left(\mathrm{GeV}^{-1}\right)$ & $4.10 \pm 3.47$ & & & 1.00 & -0.91 & -0.92 & -0.68 & -0.75 & -0.17 \\
\hline$c_{5}\left(\mathrm{GeV}^{-1}\right)$ & $-5.11 \pm 3.73$ & & & & 1.00 & 0.92 & 0.44 & 0.78 & -0.07 \\
\hline $\bar{\kappa}\left(\mathrm{GeV}^{-2}\right)$ & $-0.06 \pm 0.17$ & & & & & 1.00 & 0.41 & 0.77 & -0.19 \\
\hline $\bar{e}_{1}\left(\mathrm{GeV}^{-3}\right)$ & $2.38 \pm 1.65$ & & & & & & 1.00 & 0.39 & 0.80 \\
\hline $\bar{e}_{2}\left(\mathrm{GeV}^{-3}\right)$ & $-5.78 \pm 1.83$ & & & & & & & 1.00 & -0.11 \\
\hline$\mu(\mathrm{GeV})$ & $1.03 \pm 0.36$ & & & & & & & & 1.00 \\
\hline$\chi^{2} /$ d.o.f. & $\frac{3.90}{11-8}=1.30$ & & & & & & & & \\
\hline
\end{tabular}

\begin{tabular}{|c|c|c|c|c|c|c|c|c|c|}
\hline & Fit u2 & $c_{2}$ & $c_{3}$ & $c_{4}$ & $c_{5}$ & $\bar{\kappa}$ & $\bar{e}_{1}$ & $\bar{e}_{2}$ & $\mu$ \\
\hline$c_{2}\left(\mathrm{GeV}^{-1}\right)$ & $-3.03 \pm 1.76$ & 1.00 & -0.45 & -0.99 & 0.48 & 0.63 & 0.35 & -0.46 & -0.53 \\
\hline$c_{3}\left(\mathrm{GeV}^{-1}\right)$ & $0.96 \pm 0.98$ & & 1.00 & 0.52 & -0.98 & -0.63 & 0.45 & 0.54 & 0.69 \\
\hline$c_{4}\left(\mathrm{GeV}^{-1}\right)$ & $2.72 \pm 1.27$ & & & 1.00 & -0.55 & -0.66 & -0.28 & 0.48 & 0.56 \\
\hline$c_{5}\left(\mathrm{GeV}^{-1}\right)$ & $-0.53 \pm 1.23$ & & & & 1.00 & 0.73 & -0.46 & -0.61 & -0.78 \\
\hline $\bar{\kappa}\left(\mathrm{GeV}^{-2}\right)$ & $0.48 \pm 0.38$ & & & & & 1.00 & -0.23 & -0.95 & -0.89 \\
\hline $\bar{e}_{1}\left(\mathrm{GeV}^{-3}\right)$ & $1.29 \pm 0.38$ & & & & & & 1.00 & 0.28 & 0.41 \\
\hline $\bar{e}_{2}\left(\mathrm{GeV}^{-3}\right)$ & $-8.19 \pm 3.38$ & & & & & & & 1.00 & 0.81 \\
\hline$\mu(\mathrm{GeV})$ & $0.545 \pm 0.124$ & & & & & & & & 1.00 \\
\hline \multirow[t]{2}{*}{$\chi^{2} /$ d.o.f. } & $\frac{13.26}{16-8}=1.66$ & & & & & & & & \\
\hline & Fit u3 & $c_{2}$ & $c_{3}$ & $c_{4}$ & $c_{5}$ & $\bar{\kappa}$ & $\bar{e}_{1}$ & $\bar{e}_{2}$ & $\mu$ \\
\hline$c_{2}\left(\mathrm{GeV}^{-1}\right)$ & $-6.26 \pm 1.36$ & 1.00 & -0.83 & -0.99 & 0.83 & 0.90 & -0.83 & 0.38 & -0.83 \\
\hline$c_{3}\left(\mathrm{GeV}^{-1}\right)$ & $3.39 \pm 1.84$ & & 1.00 & 0.88 & -0.99 & -0.84 & 0.97 & -0.41 & 0.91 \\
\hline$c_{4}\left(\mathrm{GeV}^{-1}\right)$ & $5.25 \pm 1.16$ & & & 1.00 & -0.88 & -0.91 & 0.88 & 0.38 & 0.86 \\
\hline$c_{5}\left(\mathrm{GeV}^{-1}\right)$ & $-5.16 \pm 2.23$ & & & & 1.00 & 0.86 & -0.98 & 0.54 & -0.95 \\
\hline $\bar{\kappa}\left(\mathrm{GeV}^{-2}\right)$ & $-0.16 \pm 0.06$ & & & & & 1.00 & -0.84 & 0.44 & -0.92 \\
\hline $\bar{e}_{1}\left(\mathrm{GeV}^{-3}\right)$ & $2.18 \pm 0.64$ & & & & & & 1.00 & -0.52 & 0.95 \\
\hline $\bar{e}_{2}\left(\mathrm{GeV}^{-3}\right)$ & $-5.44 \pm 1.12$ & & & & & & & 1.00 & -0.61 \\
\hline$\mu(\mathrm{GeV})$ & $1.164 \pm 0.228$ & & & & & & & & 1.00 \\
\hline$\chi^{2} /$ d.o.f. & $\frac{18.37}{21-8}=1.41$ & & & & & & & & \\
\hline
\end{tabular}

should be large enough to roll over into a negative number which is estimated to be $-1.05 \mathrm{fm}$ in Ref. [20]. Thus, the iterated method is necessary to describe the channel with a bound state.

\section{B. Iterated method}

Then we determine the constants using the iterated formula, Eq. (26). The constants include the LECs $\left(c_{2,3,4,5}, \bar{\kappa}, \bar{e}_{1}, \bar{e}_{2}\right)$, and also the cutoff range scale $\mu$. The lattice data and the conventions are the same as the perturbative method, except that the lattice QCD data of the channel $D K(I=0)$ is also included from Ref. [22]. The data point near the physical value is used where $m_{\pi}=$ $156 \mathrm{MeV}$ and $m_{K}=504 \mathrm{MeV}$. For the values of the $M_{D}$, $f_{\pi}$ and $f_{K}$, we take their physical parameters from PDG, see above. The difference is $\sim 12 \%$ for $m_{\pi}$, and only $\sim 2 \%$ for $m_{K}$. Thus, the deviation from these parameters is expected to be small. We also take three different fits indicated as Fit u1, Fit u2, and Fit u3 which correspond to the data of the five channels used in the perturbative method. Therefore, there are 11, 16, and 21 data in total for Fit u1, Fit u2, and Fit u3, respectively. The resulting LECs with the correlations for the three fits can be found in Table III.

The iterated scattering lengths formula is nonlinear which can make the correlations become nonsense. However, the correlations with the uncertainties of the constants can still be used to measure the errors of the scattering lengths when the formula is linear in the local region. Fortunately, the three fits for the six channels at the lattice points all fall in the approximate linear region, and there are no unacceptable large values. The absolute value of the correlation between $c_{2}$ and $c_{4}\left(c_{3}\right.$ and $\left.c_{5}\right)$ is still very close to one, which also means that the nonlinear effect is marginal in the six channels at the lattice points. The values of the LECs are also of natural size, and better results are 

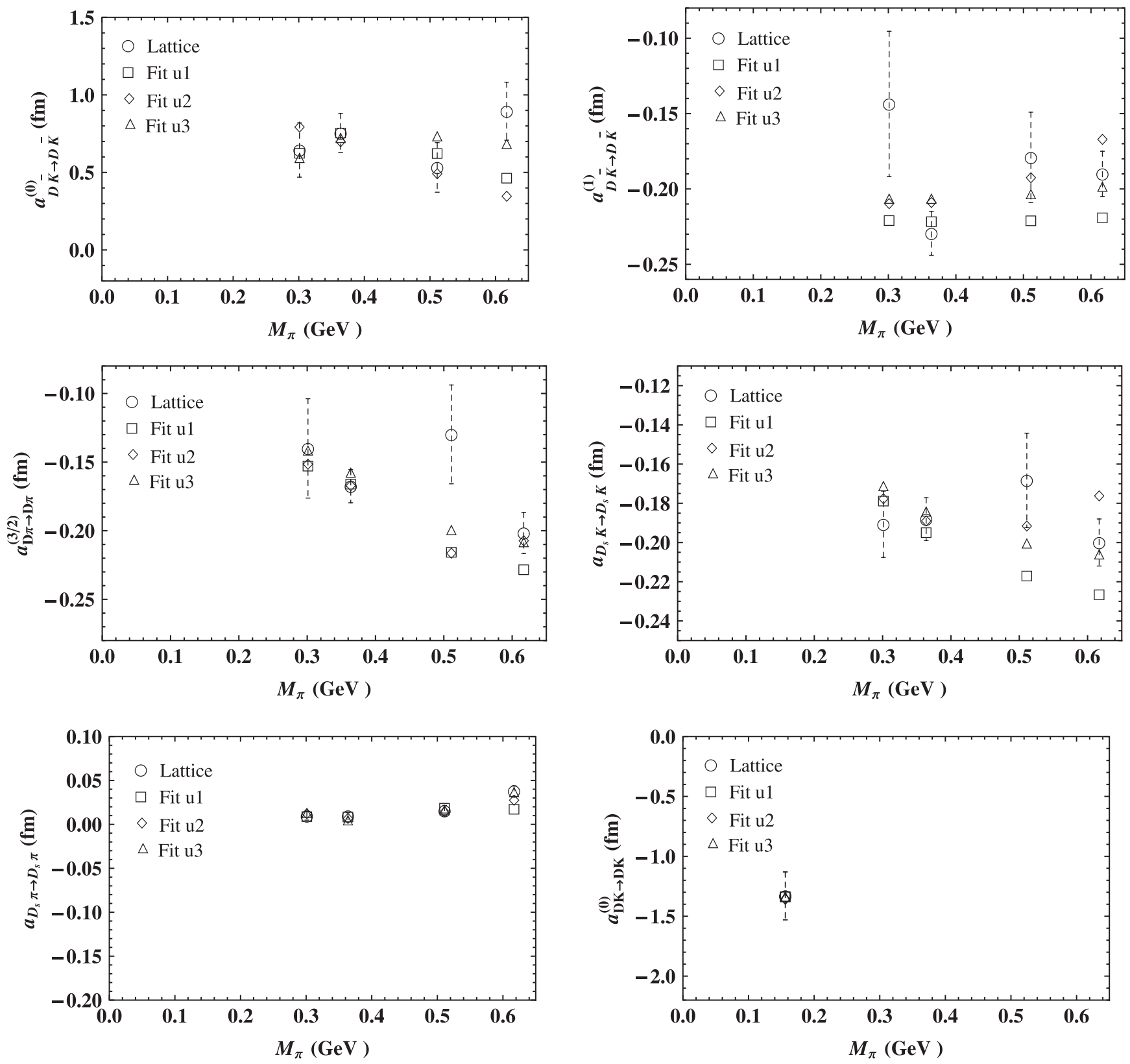

FIG. 3. Comparison of the results of the six-channel fits through the iterated formula, Eq. (26), to the lattice data of the scattering lengths from Refs. [20,22].

obtained in the three iterated fits. We present the corresponding scattering lengths from the three iterated fits in Fig. 3. We can clearly see that the results are improved significantly, especially for $m_{\pi} \simeq 511,617 \mathrm{MeV}$. In the channel $D_{s} \pi$ and $D K(I=0)$, the scattering lengths from all of the three iterated fits are in good agreement with the lattice data points. However, for the $D \bar{K}(I=1)$, the scattering length at $m_{\pi} \simeq$ $301 \mathrm{MeV}$ still has a large deviation because of the large error from the lattice data point, but they have the same order of magnitude.

After the constants are determined using the lattice data points, we make the predictions of the scattering lengths for the 11 channels at the physical values in the iterated formula. The values of the corresponding physical parameters are from PDG, see above. The results from the three iterated fits are presented in Table IV. For comparison, the values from the other two methods [20,56] are listed in the table. The errors of the scattering lengths are also estimated from the statistical errors of the constants using the error propagation formula with the correlations, as done in the perturbative method. The scattering lengths in our calculation have no imaginary parts because the contributions include all the diagrams except the iterated one-loop diagrams, and the iterated scattering length formula only involves the real part. 
TABLE IV. Predictions of the scattering lengths through the iterated formula, Eq. (26). The LECs are from the three different iterated fits, see Table III. The scattering lengths are in units of fm. For the entries marked by asterisks, only the values without uncertainties are given. See the main text for details.

\begin{tabular}{lccccc}
\hline \hline & Fit u1 & Fit u2 & Fit u3 & Liu2013[20] & Guo2019[56] \\
\hline$a_{D K}^{(1)}$ & $-0.03(4)$ & $-0.01(3)$ & $-0.06(1)$ & $0.07_{-0.03}^{+0.03}+i 0.17_{-0.01}^{+0.02}$ & $-0.01_{-0.03}^{+0.05}+i 0.39_{-0.04}^{+0.04}$ \\
$a_{D K}^{(0)}$ & $-1.55(39)$ & $-1.42(28)$ & $-1.67(45)$ & $-0.84_{-0.22}^{+0.17}$ & $-1.51_{-2.35}^{+0.72}$ \\
$a_{D \bar{K}}^{(1)}$ & $-0.23(2)$ & $-0.24(2)$ & $-0.21(1)$ & $-0.20(1)$ & $-0.20_{-0.01}^{+0.01}$ \\
$a_{D \bar{K}}^{(0)}$ & $8.76^{*}$ & $1.81(48)$ & $8.95^{*}$ & $0.84(15)$ & $21.9^{*}$ \\
$a_{D_{s} K}$ & $-0.14(3)$ & $-0.17(3)$ & $-0.14(1)$ & $-0.18(1)$ & $-0.20_{-0.01}^{+0.01}$ \\
$a_{D_{s} \bar{K}}$ & $0.14(36)$ & $371.58^{*}$ & $0.05(18)$ & $-0.09_{-0.05}^{+0.06}+i 0.44_{-0.05}^{+0.05}$ & $-0.57_{-0.04}^{+0.06}+i 0.35_{-0.07}^{+0.08}$ \\
$a_{D \pi}^{(3 / 2)}$ & $-0.07(4)$ & $-0.06(2)$ & $-0.05(1)$ & $-0.100(2)$ & $-0.103_{-0.003}^{+0.003}$ \\
$a_{D \pi}^{(1 / 2)}$ & $1.45(169)$ & $0.61(11)$ & $6.00^{*}$ & $0.37_{-0.02}^{+0.03}$ & $0.40_{-0.02}^{+0.03}$ \\
$a_{D_{s} \pi}$ & $0.02(3)$ & $0.03(2)$ & $0.05(2)$ & $-0.002(1)$ & $0.012_{-0.003}^{+0.003}$ \\
$a_{D \eta}$ & $-0.04(14)$ & $0.03(8)$ & $-0.09(2)$ & & $0.29_{-0.22}^{+0.15}+i 0.61_{-0.26}^{+0.30}$ \\
$a_{D_{s} \eta}$ & $-0.18(8)$ & $0.03(16)$ & $-0.19(2)$ & & $-0.39_{-0.03}^{+0.05}+i 0.06_{-0.02}^{+0.02}$ \\
\hline \hline
\end{tabular}

For most channels in the three fits, reasonable values are obtained. Unfortunately, the values from the $D \bar{K}(I=0)$ (Fit u1, u3), $D_{s} \bar{K}$ (Fit u2), and $D \pi(I=1 / 2)$ (Fit u3), marked by asterisks, are dominated by the nonlinear effect and have huge uncertainties. The reason for the nonlinear effect is that a bound-state pole can be generated by using parameters which are close to the physical values. However, the bound-state pole is physical only when all of the parameters are from the physical values. The values with the asterisks are too large to be accepted as the physical values. We can find that the values of the three channels are not all in the nonlinear region for the three fits, which indicates that the results are sensitive to the inputs. In other words, we can not really improve the results through the advanced analysis method. The more constraints, e.g., using more lattice QCD data points, are one of the most effective methods to obtain the physical scattering lengths. Except the values dominated by the nonlinear effect, most values are either small negative or positive numbers which indicate the small repulsive or attractive interactions. However, at this time the values of the channel $D K(I=0)$ for the three fits are large enough to roll over into negative numbers which are attractive enough to generate the wellknown bound state $D_{s 0}^{*}(2317)$. The result for this channel is also consistent with the one from Refs. [20,55,56] within errors. For the channels $D K(I=1)$ and $D_{s} \bar{K}$, large negative numbers were obtained in Ref. [55], which does not mean that the bound state can be generated because of the large imaginary parts.

Finally, we can estimate the meson and doubly charmed (bottom) baryon scattering lengths through the heavy diquark-antiquark (HDA) symmetry, as done in Ref. [63]. We take the values of the physical baryon masses: $M_{\Xi_{c c}}=3621.55 \mathrm{MeV}, M_{\Omega_{c c}}=3778 \mathrm{MeV}$, $M_{\Xi_{b b}}=10202 \mathrm{MeV}$, and $M_{\Omega_{b b}}=10359 \mathrm{MeV}$ from the PDG [62] and the relativistic quark model [64]. The other physical parameters and LECs are taken as above. The perturbative and iterated methods have also been used to predict the scattering lengths. The results are presented in Appendixes A (charmed baryons) and B (bottom baryons). From Tables V, VI, and VII, we can see clearly that the channel $\bar{K} \Xi_{c c}(I=0)$ has strong attraction but still not strong enough to generate any bound state. After using the iterated formula in Table VIII, the value of the scattering length for the channel $\bar{K} \Xi_{c c}(I=0)$ becomes large enough to roll over into a negative number which is attractive enough to generate a bound state. The same feature also exists in the channel $\bar{K} \Xi_{b b}(I=0)$ from Tables IX, X, XI, and XII. The other features for the mesons and doubly charmed (bottom) baryon scattering lengths are almost the same as the above pseudoscalar meson and heavy meson scattering. For comparison, we also list the meson and doubly charmed baryon scattering lengths from Ref. [65] where the unitarized chiral perturbation theory combined with the leading-order amplitudes was used in Table VIII of Ref. [65]. The uncertainties of the scattering lengths for the channel $K \Xi_{c c / b b}(I=0)$ also can become huge, which indicates that the value is also dominated by the nonlinear effect.

\section{SUMMARY}

In the past decades, many near-threshold nonconventional new hadron states were discovered, many of which contain one, two, three, or even four heavy quarks. Unlike the nucleon-nucleon interaction which has been studied very carefully for several decades, the interactions either between the pseudoscalar mesons and heavy hadrons or between two heavy hadrons are not known well. These interactions are crucial to unveiling the underlying structures of many so-called hidden-charm tetraquarks, pentaquarks, or even doubly charmed $T_{c c}$ states. 
Besides these manifestly exotic hadrons, the interpretation of the conventional hadron spectrum also requires the precise knowledge of the hadron-hadron interaction. For example, without the knowledge of the $\pi \pi, \pi \mathrm{K}$, and $\mathrm{KK}$ interactions, we are unable to really understand the lowlying scalar mesons below $1 \mathrm{GeV}$. Moreover, we all know that there exist very strong couple-channel effects in the formation of the low-lying parity-odd nucleon and hyperon excitations such as $N(1535), \Lambda(1405)$ etc. where the pseudoscalar meson and nucleon/hyperon interaction is the underlying driving force. Similarly, the strong $D K$ $S$-wave interaction leads to the strong channel coupling between the $D K$ scattering channel and the bare $c \bar{s}$ state in the quark model, which results in the physical $D_{s 0}^{*}(2317)$ state. Although there exist various denotations or terminology such as the $D K$ molecule etc. for the $D_{s 0}^{*}(2317)$, the underlying dynamics is more or less the same and the $D K$ interaction plays a pivotal role.

The scattering length provides valuable information of the hadron interaction. In this work, we have calculated the threshold $T$ matrices of the light pseudoscalar meson and heavy meson scattering to fourth order in heavy meson chiral perturbation theory. We fitted the nonphysical lattice QCD data points to determine the LECs through the perturbative and iterated methods, which led to a good description of the scattering lengths at most of the lattice QCD data points. The physical scattering lengths were obtained by extrapolating the corresponding parameters to their physical values. The values of the scattering lengths tend to be convergent at fourth order for most of the channels in the perturbative method. The value of the scattering length for the channel $D K(I=0)$, which involves the well-known bound state $D_{s 0}^{*}(2317)$, was obtained correctly in the iterated method.
Based on heavy diquark-antiquark symmetry, we have also estimated the meson and doubly charmed (bottom) baryon scattering lengths. We want to emphasize that the bound states can be generated with high probability in the channels $\bar{K} \Xi_{c c}(I=0)$ and $\bar{K} \Xi_{b b}(I=0)$ through the kaon and $\Xi_{c c / b b}$ scattering. This doubly charmed molecule state is very similar to the $D_{s 0}^{*}(2317)$ state in many aspects because of the heavy diquark-antiquark symmetry. The quantum number of this state is $I J^{P}=0 \frac{1}{2}^{-}$.

Considering the orbital excitation is typically around $400-500 \mathrm{MeV}$ in the quark model, this state may strongly couple with the P-wave $\Omega_{c c / b b}$ state with the quark content $c c s / b b s$ and $I J^{P}=0 \frac{1}{2}^{-}$. The physical state should lie slightly below the $\bar{K} \Xi_{c c / b b}$ threshold. The only kinematically allowed strong decay mode is $\Omega_{c c / b b}+\pi$, which violates the isospin symmetry. Hence its strong decay width is around $\sim 0.1 \mathrm{MeV}$ or even less. Its E1 electromagnetic decay width is comparable with its strong decay width, around $100 \mathrm{keV}$. We strongly urge the LHCb Collaboration to look for the P-wave excitations of the $\Omega_{c c / b b}$ and identify this very narrow signal in the coming future.

\section{ACKNOWLEDGMENTS}

This work is supported by the National Natural Science Foundation of China under Grants No. 11975033, No. 12070131001, and No. 12147127, and China Postdoctoral Science Foundation (Grant No. 2021M700251).

\section{APPENDIX A: MESON AND DOUBLY CHARMED BARYON SCATTERING LENGTHS}

This appendix gives Tables V-VIII.

TABLE V. Values of the mesons and doubly charmed baryons scattering lengths in the calculation of the perturbative formula, Eq. (25). The LECs are from the Fit p1 of Table I. The scattering lengths are in units of fm. Here $\Xi_{c c}=\left(\Xi_{c c}^{++}, \Xi_{c c}^{+}\right)^{T}$.

\begin{tabular}{lccccc}
\hline \hline Fit $\mathrm{p} 1$ & $\mathcal{O}(p)$ & $\mathcal{O}\left(p^{2}\right)$ & $\mathcal{O}\left(p^{3}\right)$ & $\mathcal{O}\left(p^{4}\right)$ & Total \\
\hline$a_{K \Xi_{c c}}^{(1)}$ & -0.28 & 0.20 & -0.55 & 0.37 & $-0.26(3)$ \\
$a_{K \Xi_{c c}}^{(0)}$ & 0.28 & -0.04 & 0.82 & -0.17 & $0.89(7)$ \\
$a_{\bar{K} \Xi_{c c}}^{(1)}$ & 0.00 & 0.08 & $-0.13+0.22 i$ & $0.10-0.14 i$ & $0.05(3)+0.08(18) i$ \\
$a_{\bar{K} \Xi_{c c}}^{(0)}$ & 0.56 & 0.32 & 0.86 & 0.46 & $2.20(103)$ \\
$a_{K \Omega_{c c}}$ & 0.28 & 0.20 & $0.23+0.33 i$ & $0.36+0.21 i$ & $1.07(53)+0.54(24) i$ \\
$a_{\bar{K} \Omega_{c c}}^{(3 / 2)}$ & -0.28 & 0.20 & -0.42 & 0.27 & $-0.23(8)$ \\
$a_{\pi \Xi_{c c}}^{(1 / 2)}$ & -0.12 & 0.03 & -0.04 & 0.04 & $-0.10(4)$ \\
$a_{\pi \Xi_{c c}}^{(2)}$ & 0.25 & 0.03 & 0.02 & -0.01 & $0.28(2)$ \\
$a_{\pi \Omega_{c c}}$ & 0.00 & 0.01 & -0.04 & 0.05 & $0.02(2)$ \\
$a_{\eta \Xi_{c c}}$ & 0.00 & 0.13 & $0.0+0.24 i$ & $0.02-0.11 i$ & $0.15(9)+0.13(3) i$ \\
$a_{\eta \Omega_{c c}}$ & 0.00 & 0.36 & $0.00+0.48 i$ & $0.20+0.22 i$ & $0.56(29)+0.70(14) i$ \\
\hline \hline
\end{tabular}


TABLE VI. The description is the same as Table V, except that the LECs are from the Fit p2 of Table I.

\begin{tabular}{lccccc}
\hline \hline Fit $\mathrm{p} 2$ & $\mathcal{O}(p)$ & $\mathcal{O}\left(p^{2}\right)$ & $\mathcal{O}\left(p^{3}\right)$ & $\mathcal{O}\left(p^{4}\right)$ & Total \\
\hline$a_{K \Xi_{c c}}^{(1)}$ & -0.28 & 0.24 & -0.61 & 0.39 & $-0.25(3)$ \\
$a_{K \Xi_{c c}}^{(0)}$ & 0.28 & -0.05 & 0.87 & -0.26 & $0.84(6)$ \\
$a_{\bar{K} \Xi_{c c}}^{(1)}$ & 0.00 & 0.09 & $-0.13+0.22 i$ & $0.07-0.18 i$ & $0.03(3)+0.03(9) i$ \\
$a_{\bar{K} \Xi_{c c}}^{(0)}$ & 0.56 & 0.39 & 0.97 & 0.52 & $2.45(58)$ \\
$a_{K \Omega_{c c}}$ & 0.28 & 0.24 & $0.29+0.33 i$ & $0.37+0.28 i$ & $1.19(29)+0.60(11) i$ \\
$a_{\bar{K} \Omega_{c c}}$ & -0.28 & 0.24 & -0.48 & 0.27 & $-0.25(4)$ \\
$a_{\pi \Xi_{c c}}^{(3 / 2)}$ & -0.12 & 0.03 & -0.04 & 0.04 & $-0.10(3)$ \\
$a_{\pi \Xi_{c c}}^{(1 / 2)}$ & 0.25 & 0.03 & 0.02 & -0.02 & $0.28(2)$ \\
$a_{\pi \Omega_{c c}}$ & 0.00 & 0.01 & -0.04 & 0.05 & $0.03(2)$ \\
$a_{\eta \Xi_{c c}}$ & 0.00 & 0.16 & $0.0+0.24 i$ & $0.00-0.13 i$ & $0.16(6)+0.11(1) i$ \\
$a_{\eta \Omega_{c c}}$ & 0.00 & 0.42 & $0.00+0.48 i$ & $0.22+0.26 i$ & $0.64(17)+0.73(7) i$ \\
\hline \hline
\end{tabular}

TABLE VII. The description is the same as Table V, except that the LECs are from the Fit p3 of Table I.

\begin{tabular}{lccccc}
\hline \hline Fit p3 & $\mathcal{O}(p)$ & $\mathcal{O}\left(p^{2}\right)$ & $\mathcal{O}\left(p^{3}\right)$ & $\mathcal{O}\left(p^{4}\right)$ & Total \\
\hline$a_{K \Xi_{c c}}^{(1)}$ & -0.28 & 0.13 & -0.37 & 0.27 & $-0.25(2)$ \\
$a_{K \Xi_{c c}}^{(0)}$ & 0.28 & 0.04 & 0.63 & -0.18 & $0.78(5)$ \\
$a_{\bar{K} \Xi_{c c}}^{(1)}$ & 0.00 & 0.09 & $-0.13+0.22 i$ & $0.04-0.02 i$ & $-0.00(2)+0.19(5) i$ \\
$a_{\bar{K} \Xi_{c c}}^{(0)}$ & 0.56 & 0.17 & 0.49 & 0.38 & $1.60(33)$ \\
$a_{K \Omega_{c c}}$ & 0.28 & 0.13 & $0.04+0.33 i$ & $0.28+0.03 i$ & $0.73(16)+0.36(5) i$ \\
$a_{\bar{K} \Omega_{c c}}$ & -0.28 & 0.13 & -0.23 & 0.22 & $-0.17(3)$ \\
$a_{\pi \Xi_{c c}}^{(3 / 2)}$ & -0.12 & 0.02 & -0.03 & 0.06 & $-0.08(2)$ \\
$a_{\pi \Xi_{c c}}^{(1 / 2)}$ & 0.25 & 0.02 & 0.01 & 0.02 & $0.29(1)$ \\
$a_{\pi \Omega_{c c}}$ & 0.00 & 0.01 & -0.04 & 0.06 & $0.03(1)$ \\
$a_{\eta \Xi_{c c}}$ & 0.00 & 0.10 & $0.0+0.24 i$ & $0.11-0.06 i$ & $0.21(3)+0.18(0) i$ \\
$a_{\eta \Omega_{c c}}$ & 0.00 & 0.23 & $0.00+0.48 i$ & $0.18+0.12 i$ & $0.41(9)+0.60(4) i$ \\
\hline \hline
\end{tabular}

TABLE VIII. Values of the mesons and doubly charmed baryons scattering lengths in the calculation of the iterated formula, Eq. (26). The LECs are from the three different iterated fits, see Table III. The scattering lengths are in units of fm. Here $\Xi_{c c}=\left(\Xi_{c c}^{++}, \Xi_{c c}^{+}\right)^{T}$. For the entries marked by asterisks, only the values without uncertainties are given. See the main text for details.

\begin{tabular}{lcccc}
\hline \hline & Fit u1 & Fit u2 & Fit u3 & Guo2017 [65] \\
\hline$a_{K \Xi_{c c}}^{(1)}$ & $-0.24(3)$ & $-0.25(2)$ & $-0.22(2)$ & $-0.19_{-0.02}^{+0.02}$ \\
$a_{K \Xi_{c c}}^{(0)}$ & $-6.15^{*}$ & $2.81^{*}$ & $-5.04^{*}$ & $5.2,-3.6,-1.4$ \\
$a_{\bar{K} \Xi_{c c}}^{(1)}$ & $-0.04(4)$ & $-0.01(3)$ & $-0.06(2)$ & $-0.22_{-0.14}^{+0.14}+i 0.45_{-0.09}^{+0.00}$ \\
$a_{\bar{K} \Xi_{c c}}^{(0)}$ & $-1.19(21)$ & $-1.29(24)$ & $-1.20(19)$ & $-0.49_{-0.19}^{+0.10}$ \\
$a_{K \Omega_{c c}}$ & $0.16(43)$ & $-7.80^{*}$ & $0.06(20)$ & $-0.55_{-0.16}^{+0.11}+i 0.13_{-0.07}^{+0.19}$ \\
$a_{\bar{K} \Omega_{c c}}^{(3 / 2)}$ & $-0.15(3)$ & $-0.18(3)$ & $-0.15(1)$ & $-0.19_{-0.02}^{+0.02}$ \\
$a_{\pi \Xi_{c c}}^{(1 / 2)}$ & $-0.07(3)$ & $-0.07(2)$ & $-0.05(1)$ & $-0.095_{-0.004}^{+0.003}$ \\
$a_{\pi \Xi_{c c}}$ & $1.73^{*}$ & $0.65(12)$ & $16.26^{*}$ & $0.55_{-0.10}^{+0.16}$ \\
$a_{\pi \Omega_{c c}}$ & $0.02(3)$ & $0.03(2)$ & $0.05(1)$ & $0.03_{-0.01}^{+0.01}$ \\
$a_{\eta \Xi_{c c}}$ & $-0.05(15)$ & $0.03(10)$ & $-0.09(2)$ & $-0.72_{-0.17}^{+0.21}+i 0.30_{-0.18}^{+1.10}$ \\
$a_{\eta \Omega_{c c}}$ & $-0.19(8)$ & $0.04(18)$ & $-0.20(2)$ & $-0.26_{-0.03}^{+0.03}+i 0.02_{-0.01}^{+0.02}$ \\
\hline \hline
\end{tabular}




\section{APPENDIX B: MESON AND DOUBLY BOTTOM BARYON SCATTERING LENGTHS}

This appendix gives Tables IX-XII.

TABLE IX. Values of the mesons and doubly bottom baryons scattering lengths in the calculation of the perturbative formula, Eq. (25). The LECs are from the Fit p1 of Table I. The scattering lengths are in units of fm. Here $\Xi_{b b}=\left(\Xi_{b b}^{0}, \Xi_{b b}^{-}\right)^{T}$.

\begin{tabular}{lccccc}
\hline \hline Fit p1 & $\mathcal{O}(p)$ & $\mathcal{O}\left(p^{2}\right)$ & $\mathcal{O}\left(p^{3}\right)$ & $\mathcal{O}\left(p^{4}\right)$ & Total \\
\hline$a_{K \Xi_{b b}}^{(1)}$ & -0.31 & 0.22 & -0.60 & 0.40 & $-0.28(3)$ \\
$a_{K \Xi_{b b}}^{(0)}$ & 0.31 & -0.04 & 0.88 & -0.19 & $0.96(8)$ \\
$a_{\bar{K} \Xi_{b b}}^{(1)}$ & 0.00 & 0.09 & $-0.15+0.23 i$ & $0.11-0.15 i$ & $0.05(4)+0.08(19) i$ \\
$a_{\bar{K} \Xi_{b b}}^{(0)}$ & 0.61 & 0.35 & 0.93 & 0.50 & $2.39(112)$ \\
$a_{K \Omega_{b b}}$ & 0.31 & 0.22 & $0.25+0.35 i$ & $0.39+0.23 i$ & $1.16(57)+0.58(26) i$ \\
$a_{\bar{K} \Omega_{b b}}$ & -0.31 & 0.22 & -0.45 & 0.29 & $-0.25(9)$ \\
$a_{\pi \Xi_{b b}}^{(3 / 2)}$ & -0.13 & 0.03 & -0.04 & 0.04 & $-0.10(4)$ \\
$a_{\pi \Xi_{b b}}^{(1 / 2)}$ & 0.26 & 0.03 & 0.02 & -0.02 & $0.28(2)$ \\
$a_{\pi \Omega_{b b}}$ & 0.00 & 0.01 & -0.04 & 0.05 & $0.02(2)$ \\
$a_{\eta \Xi_{b b}}$ & 0.00 & 0.14 & $0.0+0.26 i$ & $0.02-0.12 i$ & $0.16(10)+0.14(3) i$ \\
$a_{\eta \Omega_{b b}}$ & 0.00 & 0.39 & $0.00+0.52 i$ & $0.22+0.24 i$ & $0.61(32)+0.76(16) i$ \\
\hline \hline
\end{tabular}

TABLE X. The description is the same as Table IX, except that the LECs are from the Fit p2 of Table I.

\begin{tabular}{lccccc}
\hline \hline Fit p2 & $\mathcal{O}(p)$ & $\mathcal{O}\left(p^{2}\right)$ & $\mathcal{O}\left(p^{3}\right)$ & $\mathcal{O}\left(p^{4}\right)$ & Total \\
\hline$a_{K \Xi_{b b}}^{(1)}$ & -0.31 & 0.26 & -0.66 & 0.43 & $-0.27(3)$ \\
$a_{K \Xi_{b b}}^{(0)}$ & 0.31 & -0.06 & 0.95 & -0.28 & $0.91(7)$ \\
$a_{\bar{K} \Xi_{b b}}^{(1)}$ & 0.00 & 0.10 & $-0.14+0.23 i$ & $0.07-0.20 i$ & $0.03(3)+0.04(10) i$ \\
$a_{\bar{K} \Xi_{b b}}^{(0)}$ & 0.61 & 0.42 & 1.05 & 0.57 & $2.65(63)$ \\
$a_{K \Omega_{b b}}$ & 0.31 & 0.26 & $0.31+0.35 i$ & $0.40+0.30 i$ & $1.28(32)+0.65(12) i$ \\
$a_{\bar{K} \Omega_{b b}}^{(3 / 2)}$ & -0.31 & 0.26 & -0.51 & 0.29 & $-0.27(5)$ \\
$a_{\pi \Xi_{b b}}^{(1 / 2)}$ & -0.13 & 0.03 & -0.04 & 0.04 & $-0.10(3)$ \\
$a_{\pi \Xi_{b b}}$ & 0.26 & 0.03 & 0.02 & -0.02 & $0.29(2)$ \\
$a_{\pi \Omega_{b b}}$ & 0.00 & 0.01 & -0.04 & 0.05 & $0.03(2)$ \\
$a_{\eta \Xi_{b b}}$ & 0.00 & 0.17 & $0.0+0.26 i$ & $0.00-0.14 i$ & $0.17(6)+0.12(1) i$ \\
$a_{\eta \Omega_{b b}}$ & 0.00 & 0.46 & $0.00+0.52 i$ & $0.24+0.28 i$ & $0.70(18)+0.80(8) i$ \\
\hline \hline
\end{tabular}

TABLE XI. The description is the same as Table IX, except that the LECs are from the Fit p3 of Table I.

\begin{tabular}{lccccc}
\hline \hline Fit p3 & $\mathcal{O}(p)$ & $\mathcal{O}\left(p^{2}\right)$ & $\mathcal{O}\left(p^{3}\right)$ & $\mathcal{O}\left(p^{4}\right)$ & Total \\
\hline$a_{K \Xi_{b b}}^{(1)}$ & -0.31 & 0.14 & -0.40 & 0.29 & $-0.27(2)$ \\
$a_{K \Xi_{b b}}^{(0)}$ & 0.31 & 0.05 & 0.69 & -0.19 & $0.84(5)$ \\
$a_{\bar{K} \Xi_{b b}}^{(1)}$ & 0.00 & 0.09 & $-0.14+0.23 i$ & $0.05-0.02 i$ & $-0.00(2)+0.21(5) i$ \\
$a_{\bar{K} \Xi_{b b}}^{(0)}$ & 0.61 & 0.19 & 0.53 & 0.41 & $1.74(36)$ \\
$a_{K \Omega_{b b}}$ & 0.31 & 0.14 & $0.05+0.35 i$ & $0.30+0.04 i$ & $0.80(18)+0.39(5) i$ \\
\hline & & & & & (Table continued
\end{tabular}


TABLE XI. (Continued)

\begin{tabular}{lccccc}
\hline \hline Fit $\mathrm{p} 3$ & $\mathcal{O}(p)$ & $\mathcal{O}\left(p^{2}\right)$ & $\mathcal{O}\left(p^{3}\right)$ & $\mathcal{O}\left(p^{4}\right)$ & Total \\
\hline$a_{\bar{K} \Omega_{b b}}$ & -0.31 & 0.14 & -0.25 & 0.24 & $-0.18(3)$ \\
$a_{\pi \Xi_{b b}}^{(3 / 2)}$ & -0.13 & 0.02 & -0.03 & 0.07 & $-0.08(2)$ \\
$a_{\pi \Xi_{b b}}^{(1 / 2)}$ & 0.26 & 0.02 & 0.01 & 0.02 & $0.29(1)$ \\
$a_{\pi \Omega_{b b}}$ & 0.00 & 0.01 & -0.04 & 0.06 & $0.03(1)$ \\
$a_{\eta \Xi_{b b}}$ & 0.00 & 0.11 & $0.0+0.26 i$ & $0.12-0.07 i$ & $0.23(3)+0.19(0) i$ \\
$a_{\eta \Omega_{b b}}$ & 0.00 & 0.25 & $0.00+0.52 i$ & $0.19+0.13 i$ & $0.45(9)+0.65(4) i$ \\
\hline \hline
\end{tabular}

TABLE XII. Values of the mesons and doubly bottom baryons scattering lengths in the calculation of the iterated formula, Eq. (26). The LECs are from the three different iterated fits, see Table III. The scattering lengths are in units of fm. Here $\Xi_{b b}=\left(\Xi_{b b}^{0}, \Xi_{b b}^{-}\right)^{T}$. For the entries marked by asterisks, only the values without uncertainties are given. See the main text for details.

\begin{tabular}{lccc}
\hline \hline & Fit u1 & Fit u2 & Fit u3 \\
\hline$a_{K \Xi_{b b}}^{(1)}$ & $-0.24(3)$ & $-0.27(2)$ & $-0.23(2)$ \\
$a_{K \Xi_{b b}}^{(0)}$ & $-2.84^{*}$ & $4.51^{*}$ & $-2.43^{*}$ \\
$a_{\bar{K} \Xi_{b b}}^{(1)}$ & $-0.04(4)$ & $-0.01(4)$ & $-0.07(2)$ \\
$a_{\bar{K} \Xi_{b b}}^{(0)}$ & $-1.02(18)$ & $-1.22(22)$ & $-1.00(23)$ \\
$a_{K \Omega_{b b}}$ & $0.18(49)$ & $-4.52^{*}$ & $0.06(22)$ \\
$a_{\bar{K} \Omega_{b b}}^{(3 / 2)}$ & $-0.16(3)$ & $-0.19(3)$ & $-0.16(1)$ \\
$a_{\pi \Xi_{b b}}^{(1 / 2)}$ & $-0.07(3)$ & $-0.07(2)$ & $-0.05(1)$ \\
$a_{\pi \Xi_{b b}}$ & $1.99^{*}$ & $0.68(13)$ & $2.01^{*}$ \\
$a_{\pi \Omega_{b b}}$ & $0.02(4)$ & $0.04(2)$ & $0.05(2)$ \\
$a_{\eta \Xi_{b b}}$ & $-0.05(16)$ & $0.04(11)$ & $-0.10(2)$ \\
$a_{\eta \Omega_{b b}}$ & $-0.20(8)$ & $0.04(20)$ & $-0.20(2)$ \\
\hline \hline
\end{tabular}

[1] B. Aubert et al., Observation of a Narrow Meson Decaying to $D_{s}^{+} \pi^{0}$ at a Mass of $2.32-\mathrm{GeV} / c^{2}$, Phys. Rev. Lett. 90, 242001 (2003).

[2] P. Krokovny et al., Observation of the $D_{s J}(2317)$ and $D_{s J}(2457)$ in $B$ Decays, Phys. Rev. Lett. 91, 262002 (2003).

[3] D. Besson et al., Observation of a narrow resonance of mass $2.46 \mathrm{GeV} / c^{2}$ decaying to $D_{s}^{*+} \pi^{0}$ and confirmation of the $D_{\text {sJ }}^{*}(2317)$ state, Phys. Rev. D 68, 032002 (2003); Erratum, Phys. Rev. D 75, 119908 (2007).

[4] S. Godfrey and N. Isgur, Mesons in a relativized quark model with chromodynamics, Phys. Rev. D 32, 189 (1985).

[5] S. Godfrey and R. Kokoski, Properties of $p$-wave mesons with one heavy quark, Phys. Rev. D 43, 1679 (1991).

[6] M. Di Pierro and E. Eichten, Excited heavy-light systems and hadronic transitions, Phys. Rev. D 64, 114004 (2001).

[7] Y.-B. Dai, C.-S. Huang, and H.-Y. Jin, Heavy meson spectra from relativistic B-S equations to the order 1/M, Phys. Lett. B 331, 174 (1994).
[8] T. Barnes, F. E. Close, and H. J. Lipkin, Implications of a DK molecule at $2.32 \mathrm{GeV}$, Phys. Rev. D 68, 054006 (2003).

[9] Y.-Q. Chen and X.-Q. Li, Comprehensive Four-Quark Interpretation of $D_{s}(2317), D_{s}(2457)$, and $D_{s}(2632)$, Phys. Rev. Lett. 93, 232001 (2004).

[10] F.-K. Guo, P.-N. Shen, H.-C. Chiang, R.-G. Ping, and B.-S. Zou, Dynamically generated $0+$ heavy mesons in a heavy chiral unitary approach, Phys. Lett. B 641, 278 (2006).

[11] F.-K. Guo, P.-N. Shen, and H.-C. Chiang, Dynamically generated 1+ heavy mesons, Phys. Lett. B 647, 133 (2007).

[12] H.-Y. Cheng and W.-S. Hou, B decays as spectroscope for charmed four-quark states, Phys. Lett. B 566, 193 (2003).

[13] T. E. Browder, S. Pakvasa, and A. A. Petrov, Comment on the new $D_{s}^{(*)+} \pi^{0}$ resonances, Phys. Lett. B 578, 365 (2004).

[14] V. Dmitrašinović, $D_{s 0}^{+}(2317)-D_{0}(2308)$ Mass Difference as Evidence for Tetraquarks, Phys. Rev. Lett. 94, 162002 (2005).

[15] M. E. Bracco, A. Lozea, R. D. Matheus, F. S. Navarra, and M. Nielsen, Disentangling two- and four-quark state 
pictures of the charmed scalar mesons, Phys. Lett. B 624, 217 (2005).

[16] E. van Beveren and G. Rupp, Observed $D_{s}(2317)$ and Tentative $D(2100-2300)$ as the Charmed Cousins of the Light Scalar Nonet, Phys. Rev. Lett. 91, 012003 (2003).

[17] G. S. Bali, $D_{\mathrm{sj}}^{+}(2317)$ : What can the lattice say? Phys. Rev. D 68, 071501 (2003).

[18] A. Dougall, R. D. Kenway, C. M. Maynard, and C. McNeile, The spectrum of $D_{s}$ mesons from lattice QCD, Phys. Lett. B 569, 41 (2003).

[19] J. M. Flynn and J. Nieves, Elastic s-wave $B \pi, D \pi, D K$ and $K \pi$ scattering from lattice calculations of scalar form factors in semileptonic decays, Phys. Rev. D 75, 074024 (2007).

[20] L. Liu, K. Orginos, F.-K. Guo, C. Hanhart, and U.-G. Meißner, Interactions of charmed mesons with light pseudoscalar mesons from lattice QCD and implications on the nature of the $D_{s 0}^{*}(2317)$, Phys. Rev. D 87, 014508 (2013).

[21] C. B. Lang, L. Leskovec, D. Mohler, S. Prelovsek, and R. M. Woloshyn, $D_{s}$ mesons with $D K$ and $D^{*} K$ scattering near threshold, Phys. Rev. D 90, 034510 (2014).

[22] D. Mohler, C. B. Lang, L. Leskovec, S. Prelovsek, and R. M. Woloshyn, $D_{s 0}^{*}(2317)$ Meson and D-Meson-Kaon Scattering from Lattice QCD, Phys. Rev. Lett. 111, 222001 (2013).

[23] H.-X. Chen, W. Chen, X. Liu, Y.-R. Liu, and S.-L. Zhu, A review of the open charm and open bottom systems, Rep. Prog. Phys. 80, 076201 (2017).

[24] A. Walker-Loud, H.-W. Lin, D. G. Richards, R. G. Edwards, M. Engelhardt, G. T. Fleming, Ph. Hägler, B. Musch, M. F. Lin, H. Meyer, J. W. Negele, A. V. Pochinsky, M. Procura, S. Syritsyn, C. J. Morningstar, K. Orginos, D. B. Renner, and W. Schroers, Light hadron spectroscopy using domain wall valence quarks on an asqtad sea, Phys. Rev. D 79, 054502 (2009).

[25] G. S. Bali, S. Collins, A. Cox, and A. Schäfer, Masses and decay constants of the $D_{s 0}^{*}(2317)$ and $D_{s 1}(2460)$ from $N_{f}=2$ lattice QCD close to the physical point, Phys. Rev. D 96, 074501 (2017).

[26] C. Alexandrou, J. Berlin, J. Finkenrath, T. Leontiou, and M. Wagner, Tetraquark interpolating fields in a lattice QCD investigation of the $D_{s 0}^{*}(2317)$ meson, Phys. Rev. D 101, 034502 (2020).

[27] S. Weinberg, Nuclear forces from chiral Lagrangians, Phys. Lett. B 251, 288 (1990).

[28] S. Weinberg, Effective chiral Lagrangians for nucleon-pion interactions and nuclear forces, Nucl. Phys. B363, 3 (1991).

[29] J. Gasser, M. E. Sainio, and A. Svarc, Nucleons with chiral loops, Nucl. Phys. B307, 779 (1988).

[30] E. E. Jenkins and A. V. Manohar, Baryon chiral perturbation theory using a heavy fermion Lagrangian, Phys. Lett. B 255, 558 (1991).

[31] V. Bernard, N. Kaiser, J. Kambor, and U.-G. Meißner, Chiral structure of the nucleon, Nucl. Phys. B388, 315 (1992).

[32] C. Ordóñez and U. van Kolck, Chiral Lagrangians and nuclear forces, Phys. Lett. B 291, 459 (1992).

[33] E. Epelbaoum, W. Glöckle, and Ulf-G. Meißner, Nuclear forces from chiral Lagrangians using the method of unitary transformation (i): Formalism, Nucl. Phys. A637, 107 (1998).
[34] N. Fettes and U.-G. Meißner, Pion nucleon scattering in chiral perturbation theory (II): Fourth order calculation, Nucl. Phys. A676, 311 (2000).

[35] N. Kaiser, R. Brockmann, and W. Weise, Peripheral nucleon-nucleon phase shifts and chiral symmetry, Nucl. Phys. A625, 758 (1997).

[36] R. Machleidt and D. R. Entem, Chiral effective field theory and nuclear forces, Phys. Rep. 503, 1 (2011).

[37] X.-W. Kang, J. Haidenbauer, and U.-G. Meißner, Antinucleonnucleon interaction in chiral effective field theory, J. High Energy Phys. 02 (2014) 113.

[38] D. R. Entem, N. Kaiser, R. Machleidt, and Y. Nosyk, Peripheral nucleon-nucleon scattering at fifth order of chiral perturbation theory, Phys. Rev. C 91, 014002 (2015).

[39] N. Kaiser, Density-dependent NN interaction from subsubleading chiral $3 \mathrm{~N}$ forces: Intermediate-range contributions, Phys. Rev. C 101, 014001 (2020).

[40] N. Kaiser, Chiral corrections to kaon nucleon scattering lengths, Phys. Rev. C 64, 045204 (2001); Erratum, Phys. Rev. C 73, 069902 (2006).

[41] Y.-R. Liu and S.-L. Zhu, Meson-baryon scattering lengths in HB $\chi$ PT, Phys. Rev. D 75, 034003 (2007).

[42] J. Haidenbauer, S. Petschauer, N. Kaiser, U.-G. Meißner, A. Nogga, and W. Weise, Hyperon-nucleon interaction at nextto-leading order in chiral effective field theory, Nucl. Phys. A915, 24 (2013).

[43] B.-L. Huang and Y.-D. Li, Kaon-nucleon scattering to oneloop order in heavy baryon chiral perturbation theory, Phys. Rev. D 92, 114033 (2015); Erratum, Phys. Rev. D 95, 019903 (2017).

[44] B.-L. Huang, J.-S. Zhang, Y.-D. Li, and N. Kaiser, Mesonbaryon scattering to one-loop order in heavy baryon chiral perturbation theory, Phys. Rev. D 96, 016021 (2017).

[45] B.-L. Huang and J. Ou-Yang, Pion-nucleon scattering to $\mathcal{O}\left(p^{3}\right)$ in heavy baryon $\mathrm{SU}(3)$ chiral perturbation theory, Phys. Rev. D 101, 056021 (2020).

[46] B.-L. Huang, Pion-nucleon scattering to order $p^{4}$ in SU(3) heavy baryon chiral perturbation theory, Phys. Rev. D 102, 116001 (2020).

[47] B.-L. Huang, J.-B. Cheng, and S.-L. Zhu, Peripheral nucleon-nucleon scattering at next-to-next-to-leading order in SU(3) heavy baryon chiral perturbation theory, Phys. Rev. D 104, 116030 (2021).

[48] M. B. Wise, Chiral perturbation theory for hadrons containing a heavy quark, Phys. Rev. D 45, R2188 (1992).

[49] V. Bernard, Chiral perturbation theory and baryon properties, Prog. Part. Nucl. Phys. 60, 82 (2008).

[50] Y.-R. Liu, X. Liu, and S.-L. Zhu, Light pseudoscalar meson and heavy meson scattering lengths, Phys. Rev. D 79, 094026 (2009).

[51] F.-K. Guo, C. Hanhart, and U.-G. Meissner, Interactions between heavy mesons and Goldstone bosons from chiral dynamics, Eur. Phys. J. A 40, 171 (2009).

[52] L. S. Geng, N. Kaiser, J. Martin-Camalich, and W. Weise, Low-energy interactions of Nambu-Goldstone bosons with $D$ mesons in covariant chiral perturbation theory, Phys. Rev. D 82, 054022 (2010).

[53] P. Wang and X. G. Wang, Publisher's note: Study of $0^{+}$ states with open charm in the unitarized heavy meson chiral 
approach [Phys. Rev. D 86, 014030 (2012)]; 86, 039903 (2012).

[54] M. Altenbuchinger, L. S. Geng, and W. Weise, Scattering lengths of Nambu-Goldstone bosons off $D$ mesons and dynamically generated heavy-light mesons, Phys. Rev. D 89, 014026 (2014).

[55] D.-L. Yao, M.-L. Du, F.-K. Guo, and U.-G. Meißner, One-loop analysis of the interactions between charmed mesons and Goldstone bosons, J. High Energy Phys. 11 (2015) 058.

[56] Z.-H. Guo, L. Liu, U.-G. Meißner, J. A. Oller, and A. Rusetsky, Towards a precise determination of the scattering amplitudes of the charmed and light-flavor pseudoscalar mesons, Eur. Phys. J. C 79, 13 (2019).

[57] B. Borasoy and U.-G. Meißner, Chiral expansion of baryon masses and sigma-terms, Ann. Phys. (Amsterdam) 254, 192 (1997).

[58] N. Kaiser, P. B. Siegel, and W. Weise, Chiral dynamics and the low-energy kaon-nucleon interaction, Nucl. Phys. A594, 325 (1995).
[59] J. Hofmann and M. F. M. Lutz, Open charm meson resonances with negative strangeness, Nucl. Phys. A733, 142 (2004).

[60] J. Dobaczewski, W. Nazarewicz, and P.-G. Reinhard, Error estimates of theoretical models: A guide, J. Phys. G 41, 074001 (2014).

[61] B. D. Carlsson et al., Uncertainty Analysis and Order-byOrder Optimization of Chiral Nuclear Interactions, Phys. Rev. X 6, 011019 (2016).

[62] P. A. Zyla et al., Review of particle physics, Prog. Theor. Exp. Phys. (2020), $083 \mathrm{C} 01$.

[63] L. Meng and S.-L. Zhu, Light pseudoscalar meson and doubly charmed baryon scattering lengths with heavy diquarkantiquark symmetry, Phys. Rev. D 100, 014006 (2019).

[64] D. Ebert, R. N. Faustov, V. O. Galkin, and A. P. Martynenko, Mass spectra of doubly heavy baryons in the relativistic quark model, Phys. Rev. D 66, 014008 (2002).

[65] Z.-H. Guo, Prediction of exotic doubly charmed baryons within chiral effective field theory, Phys. Rev. D 96, 074004 (2017). 